University of Zurich

Department of Economics

Working Paper Series

ISSN 1664-7041 (print)

ISSN 1664-705X (online)

Working Paper No. 97

\title{
Auctioning risk: The all-pay auction under mean-variance preferences
}

Bettina Klose and Paul Schweinzer

Revised version, December 2017 


\title{
Auctioning risk: The all-pay auction under mean-variance preferences*
}

\author{
Bettina Klose ${ }^{\natural} \quad$ Paul Schweinzer ${ }^{\S}$ \\ Friday $15^{\text {th }}$ December, 2017
}

\begin{abstract}
We analyse the all-pay auction with incomplete information and variance-averse bidders. We characterise the unique symmetric equilibrium for general distributions of valuations and any number of bidders. Variance aversion is a sufficient assumption to predict that high-valuation bidders increase their bids relative to the risk-neutral case while low types decrease their bid. Considering an asymmetric two-player environment with uniformly distributed valuations, we show that a variance-averse player always bids higher than her risk-neutral opponent with the same valuation. Utilising our analytically derived bidding functions we discuss all-pay auctions with variance-averse bidders from an auction designer's perspective. We briefly consider possible extensions of our model, including noisy signals, type-dependent attitudes towards risk, and variance-seeking preferences.
\end{abstract}

JEL C7, D7, D81, L1.

Keywords: All-pay auctions, Contests, Incomplete information, Private values, Risk-aversion, Mean-Variance preferences.

\section{Introduction}

In economic contests players make irrecoverable investments in order to increase their chances of winning a prize. As such, the nature of a bid in an all-pay auction is very

*Thanks to Olivier Bos, Thomas Giebe, Todd Kaplan, Dan Kovenock, Sergio Parreiras, and Ron Siegel for helpful comments and discussions. Schweinzer appreciates the generous hospitality of CESifo Munich, Germany, and the University of Adelaide, Australia. ${ }^{\natural}$ University of Technology Sydney, Economics Discipline Group, 15 Broadway, Ultimo NSW 2007, Australia, Bettina.Klose@uts .edu.au. $\S$ Alpen-Adria-Universität Klagenfurt, 9020 Klagenfurt, Austria, paul.schweinzer@aau .at. 
similar to choosing a lottery. A high bid corresponds to lower payoffs in both the states of winning and losing, but increases the likelihood of the positive state. Alternatively, a low bid yields a lower probability of winning, but payoffs in both states are higher. The all-pay auction is therefore a game of risky choices. ${ }^{1}$ Nevertheless, typical analyses of all-pay auctions with private values focus on risk-neutral players, i.e., the maximisation of expected payoffs. ${ }^{2}$

It is known that risk-aversion systematically alters bidding behaviour in auctions and contests. Risk-averse bidding in winner-pay auctions has been analysed both with private values (e.g., Riley \& Samuelson, 1981; Maskin \& Riley, 1984; Matthews, 1987) and with complete information (e.g., Hillman \& Samet, 1987; Esö \& White, 2004; Fibich \& Gavious, 2003). A bidder in a (first-price) winner-pay auction controls, through her bid, both the probability of winning and the amount she wins. A risk averse bidder is willing to sacrifice some of this payoff (the individual value minus her bid) for a higher probability of winning (through a higher bid). Hence, in a first-price, winner-pay auction, risk aversion causes an increase in equilibrium bids relative to the risk neutral case.

In all-pay auctions, in addition, increasing one's bid has the direct negative effect of increasing the certain payment independently of both other effects. In consequence, a low valuation bidder under risk aversion wants to decrease her losses while a risk averse, high-valuation bidder wants to increase her probability of winning through more aggressive bidding in the all-pay auction. The all-pay auction with complete information has been solved in a formulation that is sufficiently general to account for risk-aversion (Siegel, 2009). Chen et al. (2017) allow for heterogeneously risk-averse bidders in a complete information all-pay auction. Nevertheless, the bidding functions of risk-averse players in the all-pay auction with incomplete information have not been found to date. Fibich et al. (2006) use asymptotic techniques to approximate players' bidding function in the limit as their utility functions approach linearity. Parreiras \& Rubinchik (2010) develop a technique to identify whether or not a particular strategy can be part of the equilibrium support or not. Consequently they study drop out and "all-or-nothing" strategies in all-pay auctions with three or more heterogeneously risk-averse players and private values drawn independently from distinct distributions.

Mean-variance analysis (Markowitz, 1952) has long been successfully applied to portfolio choice investment problems where asset managers evaluate alternative portfolios on the basis of the mean and variance of their return. It therefore may be surprising that the mechanism design literature and, specifically, the large literature on

${ }^{1}$ Leininger $(2000$, p. 2) presents a similar lottery-based interpretation of a bid in an all-pay auction.

2 This analysis coincides with expected utility maximisation if valuations and bids are measured in utility units and utility is quasi-linear. 
auctions has not yet employed this tool for studying the decision making problem of players in risky environments. Markowitz (1959) shows that mean-variance preferences approximately maximise expected utility for a wide variety of concave utility functions. Technically, whenever a second-order Taylor approximation does well over the relevant range of returns, mean-variance analysis approximates expected utility closely. Hence, mean-variance analysis is useful even when the true underlying utility function is unknown. This method is known in the literature as "implicit expected utility maximisation" (compare Markowitz, 2014). If a utility function is available explicitly, Levy \& Markowitz (1979) show that mean-variance analysis is typically accurate ${ }^{3}$ with the benefit of being more convenient and economical.

In the present paper, we attempt to close the aforementioned gap in the literature by characterising bidding and revenue-optimal sales behaviour in the all-pay auction with private values and risk-averse bidders. This auction type may be viewed as a natural candidate for an analysis that incorporates attitudes towards risk because it exposes a bidder to the inherent risk of either winning the object (potentially at a bargain) or losing one's bid without gaining anything. Employing mean-variance preferences - with the understanding that these will closely approximate expected utility maximisation for a large class of von-Neumann-Morgenstern utility functions - allows us to derive closed form solutions for the equilibrium bidding functions. These can then be used to perform comparative statics and analyse revenue.

\section{Literature}

To the best of the authors' knowledge there are no existing papers which analyse auctions or contests under mean-variance preferences. ${ }^{4}$ Papers relating to the analysis of risk aversion in general winner-pay auction environments include Maskin \& Riley (1984) and Matthews (1987), both discussing risk-averse bidders' behaviour in auctions. Further fundamental contributions include Esö \& White (2004) wo analyse precautionary bidding in auctions and Esö \& Futó (1999) who derive the revenue-optimal strategy for a risk-averse seller. Hu et al. (2010) discuss the implications of introducing reserve

\footnotetext{
3 The standard exception is exponential utility with extreme levels of risk-aversion. Such a preference implies that agents choose a small but certain gain over a "blank check," a 50-50 lottery of either breaking even or a huge gain. However, Markowitz et al. (1994) do not find any evidence for the existence of such preferences in their sample. Moreover, Simaan (1993) shows that even with highly risk-averse players, mean-variance analysis approximates expected utility well whenever a risk-free asset is available. (In the all-pay auction, the possibility of submitting a bid of zero ensures this availability of a risk-free asset.)

${ }^{4}$ It may be worth pointing out that the quadratic expected utility analysis of risk-aversion in Lazear \& Rosen (1981) also boils down to a mean-variance preference analysis as their output noise term is fully characterised by the mean and variance of the Normal distribution.
} 
prices. The existing analyses of asymmetric auctions, for instance Amann \& Leininger (1996), Lizzeri \& Persico (2000), Maskin \& Riley (2000), Fibich et al. (2004), Parreiras \& Rubinchik (2010), Kirkegaard (2012), or Kaplan \& Zamir (2012), typically employ asymmetric distributions (or supports) while we use our idiosyncratic variance-aversion parameter. Next to Parreiras \& Rubinchik (2010), to the best of the authors' knowledge, this is the only paper to analyse bidding in a contest when players are asymmetric in their degree of risk-aversion. Chen et al. (2017) compare heterogeneity in risk aversion to heterogeneity in valuations in their analysis of the complete information all-pay auction. They find that heterogeneously risk averse players typically participate in the auction while a similar heterogeneity in valuations precludes low-valuation players form bidding. Moreover, they show that further increasing the risk-aversion of the more risk averse player may result in non-monotonic total expected efforts.

In terms of revenue and payoff analysis, Matthews (1987) compares payoffs for risk averse behaviour when bidders exhibit constant and increasing absolute risk aversion (CARA and IARA, respectively). For CARA, he finds that bidders are indifferent between first- and second-price auctions, while for IARA bidders prefer the first-price auction. Smith \& Levin (1996) show that this ranking can be reversed under decreasing absolute risk aversion.

Most existing work on risk aversion in contests applies to full information Tullock contests. ${ }^{5}$ Several papers study the implications of introducing risk-aversion into this rent-seeking case. Konrad \& Schlesinger (1997) compare the differing implications of risk aversion on rent-seeking contests which allow players to increase the probability of obtaining a rent with those models in which contestants can increase the rent itself. Treich (2010) derives "prudency" conditions which ensure that risk-aversion and risk decrease efforts in rent-seeking contests. ${ }^{6}$ A more general analysis in terms of risk aversion of the same setup is Cornes \& Hartley (2012b) who focus on existence questions of both symmetric and asymmetric Nash equilibria (for the case of loss aversion see Cornes \& Hartley, 2012a; Mermer, 2013).

Despite similarities of the Tullock contest with complete information and the all-

\footnotetext{
${ }^{5}$ An analysis of the (repeated) full information case for more general success probabilities is Ireland (2004).

6 Sahm (2017) generalises this result by specifying a sufficient condition on comparative downside risk aversion under which a higher common level of risk aversion leads to lower aggregate effort in symmetric two-player Tullock contests. Robson (2012) uses a mean-variance model of preferences to confirm Treich's (2010) "irrelevance result" in the sense that for two-player Tullock contests, bidding behaviour is not affected by the introduction of an aversion to variance. We can interpret this paper as another instance of mean-variance analysis representing a close approximation of risk-averse behaviour in contests.
} 
pay auction with private values under risk-neutrality, ${ }^{7}$ the effects of risk aversion differ systematically in these two contest formats. In the former, the risk enters solely exogenously via the noise of the contest success function. Treich (2010) argues that the "game is indeed much like a raffle" and shows a "systematic negative effect of risk aversion." The contest success function in the all-pay auction is, by contrast, deterministic. Hence, risk enters the model endogenously. In the complete information case, the players' probability of winning is determined by the mixed strategies that are employed in equilibrium. Without full information, risk arises in a similar manner even in symmetric pure strategy equilibrium, because ad interim players are uncertain about the realized valuations of their opponents, which determine the contest winner.

The only existing works on risk aversion for the incomplete information all-pay auction of which we are aware are Fibich et al. (2006) and Parreiras \& Rubinchik (2010). ${ }^{8}$ In contrast to our fully analytical approach Fibich et al. (2006) show that, usually, an analytic characterisation of equilibrium strategies cannot be obtained for risk-averse players. For their main results, they turn to perturbation analysis to obtain revenue rankings for the case that players are "almost" risk neutral. Esö \& White (2004) show that under special conditions on valuations, decreasingly absolute risk averse players prefer the first-price auction to the all-pay auction. Fibich et al. (2006) extend this ranking to the case of general risk aversion for independent valuations. Their results are limited, however, by the fact that they cannot generally obtain analytic forms of the equilibrium bidding strategies of risk averse players. We can overcome this limitation at the price of focusing attention to the class of (linear) mean-variance preferences.

Parreiras \& Rubinchik (2010) analyse bidding behaviour in contests where three or more players draw their valuations from distributions with asymmetric supports and may have asymmetric attitudes toward risk. They find that these ex-ante asymmetries may lead to player drop-out or, for sufficiently risk-averse players, the use of discontinuous "all-or-nothing" strategies. Thus, both cases exhibit behaviour which is very different from the standard ex-ante symmetric equilibrium case. Although the authors cannot explicitly determine the equilibrium bidding functions in general, they construct a simple check for whether or not a particular bid can be part of a player's equilibrium strategy.

\footnotetext{
${ }^{7}$ Fu \& Lu (2012) draw a link between incomplete information models à la Lazear \& Rosen (1981) and Tullock-style rent-seeking games by designing a distribution for an additive error term which recreates the rent-seeking structure.

${ }^{8}$ Cingottini \& Menicucci (2006) study an environment composed of ex-ante symmetric bidders sharing the same preferences exhibiting constant absolute risk aversion. They find that it is revenue-optimal for the seller to exclude all but two randomly chosen competitors. Their result, which is contrary to the monotonicity of revenue in the risk neutral case, is obtained provided that bidders are either highly risk averse or very likely to possess a particular, known valuation. We generalize this finding in our environment.
} 
This test is used to establish the above participation conclusions.

The present paper deals with variance aversion which, in general, is different from risk aversion. A recent survey and detailed discussion of the differences between these approaches (from a more applied perspective) is presented by Markowitz (2014). Markowitz argues that it is a mistake to assert that the application of mean-variance analysis assumes normal (Gaussian) return distributions or quadratic utility functions. "In particular, if you believe that rational decision making may be characterized by axioms which imply that one should maximise expected utility using probability beliefs where objective probabilities are not known (as Savage (1954) persuaded many including me), then the necessary and sufficient condition for the practical use of mean-variance analysis is that a careful choice from a mean-variance efficient frontier will approximately maximise expected utility for a wide variety of concave (risk-averse) utility functions." He presents a large body of (predominantly empirical) research from six decades of mean-variance analysis in support of this view.

The theoretical literature concerned with contrasting and reconciling the two approaches - namely expected utility maximisation (EU) as axiomatized by von Neumann \& Morgenstern (1944) and Savage (1954) and mean-variance preferences (Markowitz, 1952 ) - is manifold. One of the most commonly known results is that expected utility reduces to a function of the return's mean and variance if the employed utility function is quadratic. Hence, for any distributions the rankings based on EU with quadratic utility coincide with those based on the corresponding mean-variance function (Tobin, 1958). This feature of quadratic utility functions is indeed unique (Baron, 1977; Chipman, 1973). The limitations of quadratic utility are however inherent, in particular all possible outcomes (not just their means) must be bounded to stay within the range of positive marginal utility and absolute risk aversion is increasing over this range. The assumption of quadratic utility can be avoided when the choice set, i.e., the class of asset distributions, can be restricted. A common assumption is that all assets under consideration are normally distributed. In this case, Allais (1953) provides the necessary and sufficient condition that the mean-variance function $V\left(\mu, \sigma^{2}\right)$ satisfies the partial differential equation $2 \frac{\partial V}{\partial \sigma^{2}}=\frac{\partial^{2} V}{\partial \mu^{2}}$ under which a utility function exists that equates the EU and mean-variance approaches. Chipman (1973) shows that, with normally distributed assets for every concave utility function that is bounded by $|u(x)|<a \exp \left(b x^{2}\right), a, b>0$, there exists a corresponding mean-variance function. ${ }^{9}$ The assumption of normally distributed returns is problematic because this class is not closed under probability mixing, however Baron (1977) provides support for excluding

${ }^{9}$ Liu (2004) provides a foundation of mean-variance analysis under the assumption of normality of asset distributions and simplified coarse utility, i.e., approximate probabilities. 
probability mixtures and restricting choices to pure strategies or portfolios. Ingersoll (1987) claims that elliptical distributions similarly equate mean-variance analysis and EU, but Liu (2004) points out that not all elliptical distributions have finite mean and variance and therefore the statement can only hold for median-dispersion analysis in its full generality. Tobin (1958) states that indifference curves between $\mu$ and $\sigma^{2}$ would be justified under the assumption of some two-parameter family of probability distributions. However, Rothschild \& Stiglitz (1970) argue that this formulation is confusing and cannot hold so broadly. In the same argument, Rothschild and Stiglitz also note that "all distributions which differ only by location parameters form a two parameter family." Mayer (1987) shows that there exists a mean-variance function which yields the same ranking as EU, if all distributions differ only by location and scale parameters, and argues that this condition holds in many economic models. ${ }^{10}$ The previously discussed normal distributions are clearly a special case of this result (Johnstone \& Lindley, 2013).

In our analysis of the all-pay auction, the distributions are restricted by the rules of the game. In particular, a player chooses from a set of two-point distributions, which are further restricted in that the two outcomes in the support always differ by the player's valuation. Hence, distributions in this family are fully characterized by two parameters. ${ }^{11}$ Nevertheless, in our application the distributions in a player's choice set do not only differ by parameters of location and scale.

Baron (1977) further argues that mean-variance analysis can still be consistent with EU even when neither the utility function nor the distributions are restricted in a way that guarantees coinciding rankings. Specifically, when only the optimal choice matters rather than the entire ranking, it is generally possible to find a utility function whose indifference curve is tangential to the efficient frontier in the same point as the level curve of the mean-variance function. In our analysis, players select their best-responses using a mean-variance function, thus full rankings matter at most off the equilibrium path. Furthermore, Baron (1977) argues that violations of the independence axiom (von Neumann \& Morgenstern, 1944) can be avoided when probability mixtures are excluded from the efficient frontier of the choice set. Our analysis, consistent with the literature on all-pay auctions with private values, naturally restricts attention to pure strategy equilibria and in this sense avoids conflicts with the independence axiom.

Similarly, Hanoch \& Levy (1970) suggest to exclude all distributions that are first

\footnotetext{
10 Two cumulative distribution functions $F$ and $G$ are said to differ only by location and scale parameters $\alpha$ and $\beta$ if $G(x)=F(\alpha+\beta x)$ for all $x$ with $\beta>0$.

11 Generally, two-point distributions have three degrees of freedom, but the property that for any given valuation the winning and losing payoffs in the all-pay auction differ by the same amount for any bid reduces the number of degrees of freedom to two.
} 
order stochastically dominated (f.o.s.d.) in a first step prior to a mean-variance analysis with the result of avoiding f.o.s.d. violations under mean-variance selection. It is a common practise in the analysis of both auctions and contests to restrict bidders' choice sets in a first step from the feasible set of all non-negative bids to the compact set of non-negative bids that are no higher than the bidder's valuation of winning. Generally, bids above a player's valuation are dominated in the sense of f.o.s.d. by bidding zero.

More recently, the theoretical literature has diverted attention from its previous focus on the comparison of EU and mean-variance analysis. Nakamura (1970) provides seven preference-based axioms that are necessary and sufficient for the existence of a mean-variance function representing a preference relation. Nakamura further derives specific axiomatisations for the four most common additively separable mean-variance functions including the linear specification that we employ in this paper. Qu (2017) argues that multiple experimental studies have shown systematic violations of EU (including the Allais paradox) that are nevertheless consistent with some mean-variance preferences. Qu develops a mean-variance axiomatisation in an Anscombe-Aumann setting with subjective probabilities that does not necessarily conform to EU. Making use of constant absolute uncertainty aversion, Qu also axiomatises the linear specification, "which is the most widely used mean-variance form" (Qu, 2017) and also applied in this paper.

\section{The model}

There is a seller with one indivisible object for sale. The seller's valuation of the item is zero. There are $n \geq 2$ potential buyers with valuations $\theta_{i}, i \in \mathcal{N}=\{1,2, \ldots, n\}$, respectively. The own valuation is private information of each buyer and all players' valuations, $\theta_{i}, i \in \mathcal{N}$, are assumed to be independent draws from the same absolutely continuous distribution $F$. Let $f(\cdot)=F^{\prime}(\cdot)$ represent the associated probability density function with support $[0,1]$.

After realising their own valuations of the object, $\theta_{i}$, all players simultaneously submit their bids, $b_{i}, i \in \mathcal{N}$. The player with the highest bid receives the object and all players forgo their bids. Player $i$ 's payoff is hence given by

$$
\pi\left(b_{i}, b_{-i} ; \theta_{i}\right)= \begin{cases}\theta_{i}-b_{i} & \text { if } b_{i}>b_{j} \forall j \neq i, \\ \frac{1}{m} \theta_{i}-b_{i} & \text { if } i \in Q=\left\{j \in \mathcal{N} \mid b_{j}=\max _{k \in \mathcal{N}} b_{k}\right\}, m=|Q|, \\ -b_{i} & \text { if } \exists j: b_{i}<b_{j} .\end{cases}
$$

Therefore, for a player with valuation $\theta$ each bid $b \geq 0$ corresponds to a lottery $\mathcal{L}_{\theta}(b) \equiv$ $\{(-b ; 1-\mathbb{P}\{$ win $\mid b\}),(\theta-b ; \mathbb{P}\{$ win $\mid b\})\}$, where the probability of winning $\mathbb{P}\{$ win $\mid b\}$ is 
in equilibrium determined jointly by the type distribution $F$ and the equilibrium bidding strategies.

When buyers have mean-variance preferences, they maximise an objective function $u_{i}\left(b_{i} ; \theta_{i}\right)$, which is increasing in the expected payoff, $\mu\left(b_{i} ; \theta_{i}\right) \equiv \mathbb{E}_{b_{-i}}\left(\pi\left(b_{i}, b_{-i} ; \theta_{i}\right)\right)$, and decreasing in the variance of their payoff, $\sigma^{2}\left(b_{i} ; \theta_{i}\right) \equiv \mathbb{V}_{b_{-i}}\left(\pi\left(b_{i}, b_{-i} ; \theta_{i}\right)\right)$. For our analytical investigation we use the following simple linear representation of meanvariance preferences ${ }^{12}$

$$
u_{i}\left(b ; \theta_{i}, \nu_{i}\right)=\mu\left(b ; \theta_{i}\right)-\nu_{i} \sigma^{2}\left(b ; \theta_{i}\right)
$$

in which the parameter $\nu_{i} \in[0,1 / 2]$ accounts for player $i$ 's variance-aversion. ${ }^{13}$ The case of $\nu_{i}=0$ represents the standard case of risk-neutral expected payoff maximisation. Bounding the degree of variance aversion from above guarantees the existence of a pure strategy equilibrium.

Provided a player's knowledge of her own type $\left(\theta_{i}, \nu_{i}\right)$, her bid can be interpreted as choosing a lottery with two possible outcomes. If the player submits the highest bid, she will receive her valuation of the prize $\theta_{i}$ minus the cost of her bid. In all other events, she will lose her bid. Note that the payoff difference between these two outcomes remains fixed for any bid and is just equal to the pre-determined player's valuation $\theta_{i}$. However, selecting a higher bid does not only decrease the respective payoffs for both outcomes, but also moves probability mass from the losing to the winning outcome.

In section 5 we discuss various ways to generalise this model. We introduce exogenous noise to the model through independent shocks on the winning and losing outcomes. In this case, $\theta$ is merely a noisy signal of a player's true valuation for the prize which will only be realised after the contest. We further consider the effects of variance seeking behaviour in contests, i.e., we consider negative values of $\nu_{i}$ in (1). Based on these results we briefly discuss an extension of our model that accounts for type-dependent risk preferences. That is, ex-ante symmetric players have private information about their type $\left(\theta_{i}, \nu_{i}\right)$, where $\nu_{i}\left(\theta_{i}\right)$ follows a common, specific functional form known to the players.

\footnotetext{
${ }^{12}$ A large body of empirical and theoretical work employs variants of this simple form on the basis of both tractability and testability. Multiple axiomatisations of this form exist starting from Pollatsek \& Tversky (1970) to more recent ones by Nakamura (1970) and Qu (2017). For discussions see, for instance, Tsiang (1972), Coyle (1992), Saha (1997) or the textbook treatment in Sargent \& Heller (1987, pp. 154-5). Recently, Chiu (2010) discusses the applicability of mean-variance preferences to a large set of problems in finance and economics in choice theoretic terms. In the appendix, we discuss possible generalisations of this objective function which preserve our results qualitatively.

13 In section 5.3 we extend the range of admissible values of $\nu$ to $[-1 / 2,+1 / 2]$.
} 


\section{Bidding behaviour of $n$ symmetric bidders}

In this section, we consider an all-pay auction with $n$ ex-ante symmetric players. In particular, we assume that all players have identical degrees of variance-aversion, $\nu \equiv$ $\nu_{1}=\cdots=\nu_{n}$. Therefore, a player's type $\left(\theta_{i}, \nu_{i}\right)$ is now fully characterised by her valuation $\theta_{i}$. In the first-price, all-pay auction, a type- $\theta_{i}$ bidder's expected payoff when issuing a bid of $b$ is given by

$$
\pi\left(b, \beta ; \theta_{i}\right)=\int_{0}^{\beta^{-1}(b)} \cdots \int_{0}^{\beta^{-1}(b)} \theta_{i} f\left(\theta_{1}\right) \cdots f\left(\theta_{n-1}\right) d \theta_{1} \cdots d \theta_{n-1}-b
$$

if all other $n-1$ players use an invertible bidding function $\beta(\theta)$.

As a benchmark, consider the risk neutral case, i.e., $\nu_{i}=0$ for all $i \in \mathcal{N}$. In this case, players simply maximise their expected payoffs and it is well known, for instance from Milgrom (2004, p. 119), that the strategies

$$
\beta_{\mathrm{rn}}(\theta)=\theta(F(\theta))^{n-1}-\int_{0}^{\theta}(F(\vartheta))^{n-1} d \vartheta
$$

constitute the unique symmetric equilibrium.

With mean-variance preferences, symmetric players with $\nu \in[0,1 / 2]$ choose bids which maximise (1), taking into account their payoff variance in addition to their expected payoff. These are given for the first-price, all-pay auction as

$$
\begin{aligned}
\mu\left(b ; \theta_{i}\right) & =\theta_{i} \mathbb{P}\{\text { win } \mid b\}-b \\
\sigma^{2}\left(b ; \theta_{i}\right) & =\mathbb{P}\{\operatorname{win} \mid b\}\left(\theta_{i}-b-\mu\left(b ; \theta_{i}\right)\right)^{2}+(1-\mathbb{P}\{\text { win } \mid b\})\left(-b-\mu\left(b ; \theta_{i}\right)\right)^{2} \\
& =\mathbb{P}\{\operatorname{win} \mid b\}(1-\mathbb{P}\{\operatorname{win} \mid b\}) \theta_{i}^{2}
\end{aligned}
$$

where $\mathbb{P}\{$ win $\mid b\}$ denotes the probability of the event that a player wins conditional on bidding $b{ }^{14}$ In this environment, we can solve for a non-decreasing bidding function $\beta_{m v}(\theta)$, which constitutes a symmetric equilibrium.

As illustrated in Figure 1, level curves of our mean-variance function (1), plotted grey, are generally linear upward sloping lines (with slope $\nu$ ) in $\left(\mu, \sigma^{2}\right)$-space. Each feasible lottery $\mathcal{L}_{\theta}(b), b \geq 0$, corresponds to a point $\left(\mu(b, \theta), \sigma^{2}(b, \theta)\right)$ in this $\left(\mu, \sigma^{2}\right)$-space. For a player with valuation $\theta$ the feasible set in $\left(\mu, \sigma^{2}\right)$-space is $\mathcal{F}_{\theta}=$ $\left\{\left(\mu, \sigma^{2}\right) \mid \mu=\mu(b, \theta) \wedge \sigma^{2}=\sigma^{2}(b, \theta)\right.$ for some $\left.b \geq 0\right\}$. The efficient frontier $\mathcal{E}_{\theta} \equiv$ $\mathcal{F}_{\theta} \backslash\left\{\left(\mu, \sigma^{2}\right) \mid \exists\left(\mu_{*}, \sigma_{*}^{2}\right) \in \mathcal{F}_{\theta}, \mu_{*}>\mu \wedge \sigma_{*}^{2} \leq \sigma^{2}\right.$ or $\left.\mu_{*} \geq \mu \wedge \sigma_{*}^{2}<\sigma^{2}\right\}$ is constructed

\footnotetext{
${ }^{14}$ Note that, in our model, using the modified mean-variance approach due to Blavatskyy (2010) would lead to qualitatively similar results since the mean absolute semideviation is $r\left(b, \theta_{i}\right)=$ $\left(F\left(\beta^{-1}(b)\right)\right)^{n-1}\left(1-\left(F\left(\beta^{-1}(b)\right)\right)^{n-1}\right) \theta_{i}$.
} 
in the usual way by excluding all points $\left(\mu, \sigma^{2}\right)$ from $\mathcal{F}_{\theta}$ which are strictly dominated by another feasible point which has strictly greater mean and no higher variance, or strictly lower variance and no lower mean. In particular, this means excluding all pairs $\left(\mu, \sigma^{2}\right)$ that correspond to bids $b>\theta$, because $(0,0) \in \mathcal{F}_{\theta}$ and $\mu(b ; \theta)<0, \sigma^{2}(b ; \theta) \geq 0$ if $b>\theta$. Moreover, let $\hat{b}$ such that $\mathbb{P}\{$ win $\mid \hat{b}\}=\frac{1}{2}$, then by continuity of $\mathbb{P}\{$ win $\mid b\}$ for every $b_{1}<\hat{b}$, there exists a $b_{2}>\hat{b}$ such that $\mathbb{P}\left\{\right.$ win $\left.\mid b_{1}\right\}=1-\mathbb{P}\left\{\right.$ win $\left.\mid b_{2}\right\}$, hence $\sigma^{2}\left(b_{1} ; \theta\right)=\sigma^{2}\left(b_{2} ; \theta\right)$, but in general $\mu\left(b_{1}, \theta\right) \neq \mu\left(b_{2}, \theta\right)$. By this argument, $\left(\mu, \sigma^{2}\right)$ corresponding to low (high) bids are not contained in the efficient frontier for players with high (low) valuations $\theta$. For players with intermediate valuations, $\left(\mu\left(b_{1}\right), \sigma^{2}\left(b_{1}\right)\right) \in \mathcal{E}_{\theta}$ for low $b_{1}$ but $\left(\mu\left(b_{2}\right), \sigma^{2}\left(b_{2}\right)\right) \in \mathcal{E}_{\theta}$ for high $b_{1}$. A player with valuation $\theta \in(0,1)$ chooses a bid $b \in \mathcal{E}_{\theta}$ that corresponds to $\left(\mu(b ; \theta), \sigma^{2}(b ; \theta)\right.$ at which her efficiency frontier has slope $\nu$, i.e., is tangential to the level curve of the objective function. Baron (1977) points out that this optimal choice coincides for all concave utility functions whose indifference curves are tangential to the efficiency frontier in this point.
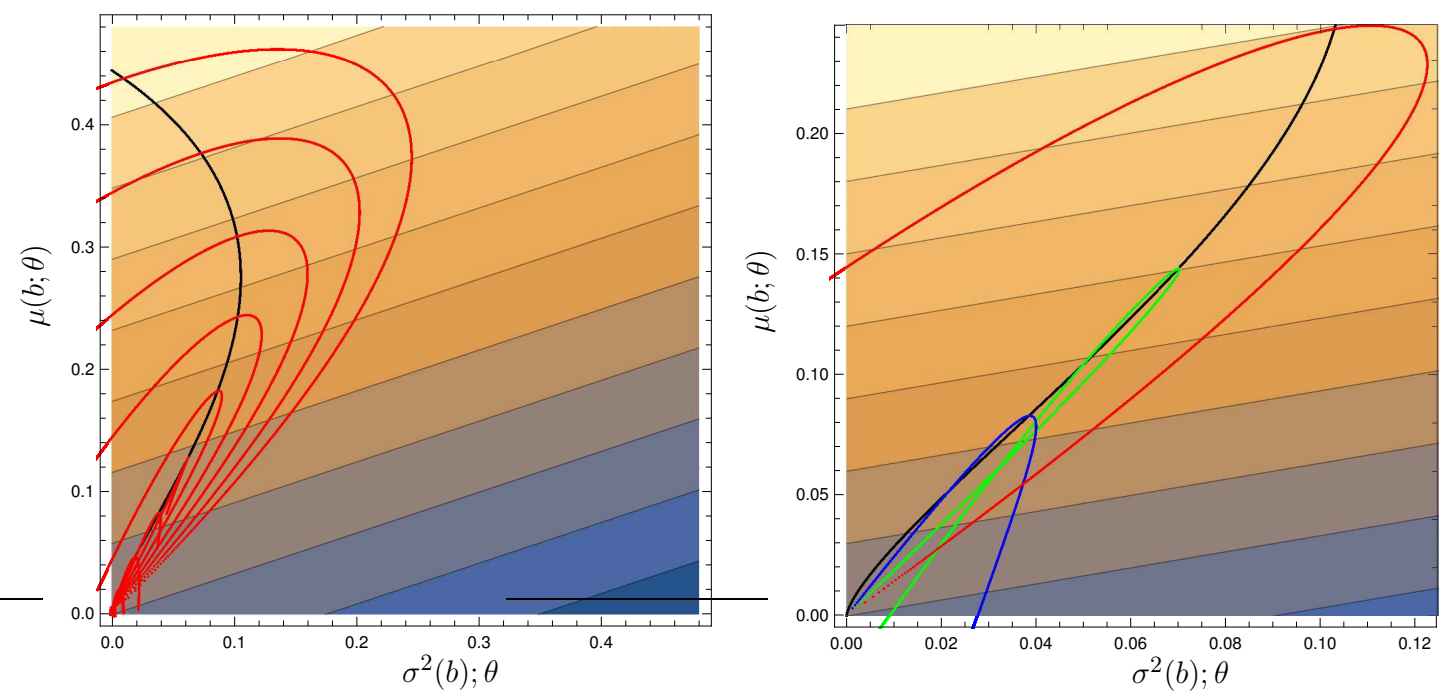

Figure 1: Level sets in $\left(\mu, \sigma^{2}\right)$-space for uniformly distributed valuations and $\nu=1 / 3$. The left-hand panel illustrates the sets of feasible pairs $\left(\mu(b ; \theta), \sigma^{2}(b ; \theta)\right)$ in red for any bid $b \in\left[0, \theta_{i}\right]$ for $\theta_{i} \in\{.1, .2 ., 3, .4, .5, .6, .7, .8, .9, .99\}$. Higher types have higher "deviation" paths. Black is the equilibrium bid for all types, intersecting the red curve at the tangency with the indifference curves. The right-hand panel zooms in on the lower-left of the left-hand panel to show the choice sets for low valuations $\{.4, .53, .7\}$ in more detail.

Proposition 1 (Symmetric Equilibrium). If $\nu_{1}=\cdots=\nu_{n}=\nu$ and players maximise the linear mean-variance function (1) ad interim, then the set of $n$ symmetric bidding 
functions

$$
\begin{aligned}
& \beta_{m v}^{1}(\theta)=\cdots=\beta_{m v}^{n}(\theta):= \\
& \beta_{r n}(\theta)-\nu(n-1) \int_{0}^{\theta} \vartheta^{2}(F(\vartheta))^{n-2} f(\vartheta)\left(1-2(F(\vartheta))^{n-1}\right) d \vartheta
\end{aligned}
$$

constitutes a symmetric equilibrium.

Proof. In order to confirm that the strategies (5) are indeed a symmetric equilibrium, we show that no profitable deviation exists. A player's utility from any bid $b$ is provided in (1). Consider a player of type $\theta$ who bids $\beta_{m v}(z), z \in[0,1]$ against opponents who bid according to $\beta_{m v}$ and their true type. ${ }^{15}$ Then, by equations (1) to (5),

$$
\begin{aligned}
u_{i}\left(\beta_{m v}(z), \theta\right)= & \theta(1-\nu \theta) F^{n-1}(z)+\nu \theta^{2} F^{2 n-2}(z)-z F^{n-1}(z)+\int_{0}^{z} F^{n-1}(\vartheta) d \vartheta \\
& +\nu \int_{0}^{z} \vartheta^{2}(n-1) F^{n-2}(\vartheta) f(\vartheta)\left(1-2 F^{n-1}(\vartheta)\right) d \vartheta .
\end{aligned}
$$

Hence, setting $z=\theta$ (i.e., bidding according to (5)) is optimal if

$$
\begin{aligned}
\frac{\partial u_{i}\left(\beta_{m v}(z), \theta\right)}{\partial z}= & \theta(1-\nu \theta)(n-1) F^{n-2}(z) f(z)+\nu \theta^{2} 2(n-1) F^{2 n-3}(z) f(z)-F^{n-1}(z) \\
& -(n-1) z F^{n-2}(z) f(z)+F^{n-1}(z) \\
& +\nu z^{2}(n-1) F^{n-2}(z) f(z)\left(1-2 F^{n-1}(z)\right) \\
= & (\theta-z)(n-1) f(z) F^{n-2}(z)\left(1-\nu(\theta+z)+2 \nu(\theta+z) F^{n-1}(z)\right)
\end{aligned}
$$

is positive for $z<\theta$, negative for $z>\theta$ and zero at $z=\theta$. This is the case if the factor $\left(1-\nu(\theta+z)+2 \nu(\theta+z) F^{n-1}(z)\right)$ is non-negative for all $(\theta, z) \in[0,1]^{2}$ and always holds if $\nu \leq 1 / 2$. It remains to be shown that $\beta_{\mathrm{mv}}$ is strictly monotone. When deriving the symmetric equilibrium bidding functions $\beta_{\mathrm{mv}}$ via the players' optimisation problem, the first-order differential equation

$$
\beta^{\prime}(\theta)=\theta\left(1-\nu \theta+2 \nu \theta(F(\theta))^{n-1}\right)(n-1)(F(\theta))^{n-2} f(\theta)
$$

is solved together with the boundary condition $\beta(0)=0$. Note that, (6) uniquely determines $\beta_{\mathrm{mv}}$, which is indeed a strictly increasing function whenever

$$
1-\nu \theta+2 \nu \theta(F(\theta))^{n-1}>0
$$

for all $\theta \in[0,1]$. This condition is equivalent to

$$
F(\theta)^{n-1}>\frac{1}{2}-\frac{1}{2 \nu \theta}, \forall \theta \in[0,1]
$$

and holds for any $F$ and $n$ if $\nu \in[0,1 / 2]$. This shows that our bidding function (5) satisfies the usual monotonicity requirement.

\footnotetext{
15 Bids below $\beta_{m v}(0)$ are not feasible and any bid $b>\beta_{m v}(1)$ is strictly dominated by $\beta_{m v}(1)$ as $b$ yields strictly lower expected payoff $\mu(b, \theta)<\mu\left(\beta_{m v}(1), \theta\right)$ without reducing the payoff variance as winning probabilities are identical under both strategies.
} 
It is now possible to see from condition (7) in the proof of proposition 1 why we need to restrict the variance-aversion parameter $\nu$. Intuitively, as players become very variance-averse, bidding zero along with the resulting certain payoff of zero becomes more attractive for players with valuations $\theta \in\left(0, \beta_{m v}(1)\right)$. Similarly bidding at the upper bound of the bid distribution and winning with certainty becomes more attractive for players with high valuations $\theta \in\left(\beta_{m v}(1), 1\right)$. This behaviour would resemble the "all-or-nothing" strategies discussed in Parreiras \& Rubinchik (2010). Therefore, in order to be able to solve for the desired strictly monotone bidding function, we need to bound the variance aversion parameter. The proof requires that $(1-\nu(\theta+z)+$ $\left.2 \nu(\theta+z) F^{n-1}(z)\right)>0$ for all $z \in(0,1), \theta \in[0,1]$, or equivalently

$$
F(z)^{n-1}>\frac{1}{2}-\frac{1}{2 \nu(z+1)} .
$$

This condition (9) generally holds if $\nu$ is sufficiently small such that $\theta \nu \leq 1 / 2$ for all $\theta$ in the support of the type distribution $F(\cdot)$, but otherwise imposes a restriction on the distribution of types and/or the number of players. ${ }^{16}$ Conversely, for a given distribution of valuations, inequality (9) illustrates how the distribution places an upper bound on the degree of variance aversion which we may consider. Interpreting the right-hand side as a c.d.f.

$$
H_{\nu}(\theta) \equiv \begin{cases}0 & \text { if } \theta \leq 1-\nu) / \nu \\ 1 / 2-1 /(2 \nu(\theta+1)) & \text { if } \theta \in((1-\nu) / \nu, \bar{\theta}) \\ 1 & \text { if } \theta>\bar{\theta}\end{cases}
$$

for some fixed large $\bar{\theta}$, condition (9) states that $H_{\nu}$ first order stochastically dominates $F^{n-1}$. As $\nu$ increases, $H_{\nu}$ increases in the sense of first order stochastic dominance and (9) fails as $\nu$ grows too large. Higher degrees of variance aversion may be considered when $F$ places sufficient mass on low types.

We would like to point out that uniqueness of the equilibrium characterised in the above proposition can be shown following the same steps that lead to Lemma 5 of Parreiras \& Rubinchik (2010) yielding uniqueness in the ex ante symmetric setting with concave von-Neumann-Morgenstern utility functions. In addition, we conjecture that Lemma 6 of Parreiras \& Rubinchik (2010) similarly implies that the only possible asymmetric equilibria in this model can consist of bidding functions exhibiting an infinite number of discontinuities.

\footnotetext{
16 The optimality condition (9) implies monotonicity of the bidding function, because

$$
\frac{1}{2}-\frac{1}{2 \nu(z+1)}>\frac{1}{2}-\frac{1}{2 \nu z}
$$
}

Hence, (9) implies (8). 
Next, taking a closer look at the equilibrium strategies described in proposition 1 , we show that low types submit lower bids under mean-variance preferences, while high types submit higher bids under mean-variance preferences than if they were to maximise expected payoff only.

Proposition 2 (Single-crossing). There exists a $\hat{\theta} \in(0,1)$ such that $\beta_{m v}(\theta) \leq \beta_{r n}(\theta)$ for $\theta \leq \hat{\theta}, \beta_{m v}(\hat{\theta})=\beta_{r n}(\hat{\theta})$ and $\beta_{m v}(\theta)>\beta_{r n}(\theta)$ for $\theta>\hat{\theta}$.

Proof. Note that the symmetric equilibrium strategy can be written as

$$
\beta_{\mathrm{mv}}(\theta)=\beta_{\mathrm{rn}}(\theta)-\nu \int_{0}^{\theta} G(\vartheta) H(\vartheta) d \vartheta
$$

where $G(\vartheta)=\vartheta^{2}(n-1)(F(\vartheta))^{n-2} f(\vartheta)$ and $H(\vartheta)=1-2(F(\vartheta))^{n-1} . F$ is a cumulative distribution function with density $f$, therefore $G(\vartheta) \geq 0$ for all $\vartheta \in[0,1]$. $H(\vartheta)$ is a continuous and decreasing function with $H(0)=1$ and $H(1)=-1$. Hence, $\int_{0}^{\theta} G(\vartheta) H(\vartheta) d \vartheta>0$ for sufficiently small $\theta>0$ and if $\int_{0}^{\hat{\theta}} G(\vartheta) H(\vartheta) d \vartheta=0$ for any $\hat{\theta}>0$, then $\int_{0}^{\theta} G(\vartheta) H(\vartheta) d \vartheta<0$ for all $\theta>\hat{\theta}$. Next, we show that $\beta_{m v}(1)>\beta_{r n}(1)$. For this we only need to show that $\int_{0}^{1} \theta^{2}(F(\theta))^{n-2} f(\theta)\left(1-2(F(\theta))^{n-1}\right) d \theta<0$.

$$
\begin{aligned}
\int_{0}^{1} & \theta^{2}(F(\theta))^{n-2} f(\theta)\left(1-2(F(\theta))^{n-1}\right) d \theta \\
= & \int_{0}^{1} \theta^{2}(F(\theta))^{n-2} f(\theta) d \theta-2 \int_{0}^{1} \theta^{2}(F(\theta))^{2 n-3} f(\theta) d \theta \\
= & \left.\theta^{2} \frac{1}{n-1}(F(\theta))^{n-1}\right|_{0} ^{1}-\int_{0}^{1} 2 \theta \frac{1}{n-1}(F(\theta))^{n-1} d \theta \\
& -2\left(\left.\theta^{2} \frac{1}{2 n-2}(F(\theta))^{2 n-2}\right|_{0} ^{1}-\int_{0}^{1} 2 \theta \frac{1}{2 n-2}(F(\theta))^{2 n-2} d \theta\right) \\
= & \frac{1}{n-1}-\frac{2}{n-1} \int_{0}^{1} \theta(F(\theta))^{n-1} d \theta-2\left(\frac{1}{2(n-1)}-\frac{1}{n-1} \int_{0}^{1} \theta(F(\theta))^{2(n-1)} d \theta\right) \\
= & \frac{2}{n-1}\left(\int_{0}^{1} \theta(F(\theta))^{2(n-1)} d \theta-\int_{0}^{1} \theta(F(\theta))^{n-1} d \theta\right) \\
< & \frac{2}{n-1}\left(\int_{0}^{1} \theta(F(\theta))^{n-1} d \theta-\int_{0}^{1} \theta(F(\theta))^{n-1} d \theta\right)=0 .
\end{aligned}
$$

The inequality follows because

$$
(F(\theta))^{2(n-1)}=(F(\theta))^{n-1} \Leftrightarrow(F(\theta))^{n-1}=0 \vee(F(\theta))^{n-1}=1
$$

and $(F(\theta))^{2(n-1)}<(F(\theta))^{n-1}$ otherwise. 
This result is qualitatively in line with propositions 1 and 2 in Fibich et al. (2006). ${ }^{17}$ Hence, our proposition 2 demonstrates that variance aversion has qualitatively the same effect on equilibrium bidding strategies as risk aversion and thus suffices to explain observed data showing such behaviour. ${ }^{18}$ The intuition behind this result is that lowvaluation bidders expect to lose in a symmetric equilibrium and therefore decrease their bids in order to keep their variance low. High-valuation bidders, by contrast, are likely to win and therefore increase their bids in line with variance compression. ${ }^{19}$ Proposition 2 implies that for any value of $\nu$, there is only a single valuation $\hat{\theta}$ at which the equilibrium bid of a player in the symmetric auction with variance-averse bidders coincides with that in the symmetric auction with risk-neutral bidders. ${ }^{20}$. From (5) it is evident that this type $\hat{\theta}$ does not depend on the variance aversion parameter $\nu$, but is fully characterised by the distribution of types, $F$, and number of players, $n$.

Our proposition 2 shows that variance aversion alone is sufficient to observe the bidding behaviour found by Fibich et al. (2006) for general risk-aversion as modelled by a utility function that is concave in payoffs. It is not obvious that the simple meanvariance approach would yield this qualitatively identical finding to the full account of all moments. The main advantage we obtain from knowing equilibrium bidding functions is our ability to further investigate the impact that variance aversion has on auction characteristics that are relevant from a designer's perspective such as revenue or bidder participation. While the general approach of Fibich et al. (2006) results in the same qualitative observations regarding the bidding function, it does not allow for such an analysis. Fibich et al. (2006) use perturbation analysis, therefore considering

17 Proposition 2 is not a special case of the results in Fibich et al. (2006). Although it is possible to model particular versions of mean-variance preferences as quadratic utility functions in the expected utility framework, this is not the case with the linear form (1).

18 Two experimental papers by Barut et al. (2002) and Noussair \& Silver (2006) explicitly test bidding behaviour in all-pay auctions with private valuations and report behaviour in line with our results. Both studies point out that risk-aversion may be a cause for the observed aggregate bidding strategy. A recent survey of the experimental literature on contests is Dechenaux et al. (2015).

19 Using (4), a bidder with type $\theta$ seeks to maximise $[\theta \mathbb{P}\{$ win $\mid b\}-b]-\nu \mathbb{P}\{$ win $\mid b\}(1-\mathbb{P}\{$ win $\mid b\}) \theta^{2}$, where $\mathbb{P}\{w i n \mid b\}$ is the probability of winning with a bid of $b$. The term in brackets coincides with the bidder's objective in a standard all-pay auction with risk neutral bidders. The second part thus explains why the bidder would want to deviate from the standard strategy. Note that $\mathbb{P}\{$ win $\mid b\}(1-\mathbb{P}\{$ win $\mid b\})$ is inversely U-shaped, with a peak at $1 / 2$. In other words, this term penalises the bidder for winning with a probability close to $1 / 2$. Thus, the bidder has a preference for extreme winning probabilities compared to the risk neutral case.

20 This distortion of bids seems to correspond to experimental evidence. Both Barut et al. (2002) and Noussair \& Silver (2006) report bidding behaviour along these lines in all-pay auctions with private valuations. Moreover, systematic differences in competition performances across genders could be explained on the basis of our (a)symmetric variance-aversion results (Gneezy et al., 2003; Niederle \& Vesterlund, 2007) 
only infinitesimally risk averse (i.e., almost risk neutral) players. ${ }^{21}$ Our analysis, by contrast, allows for preferences to exhibit a significant degree of variance aversion.

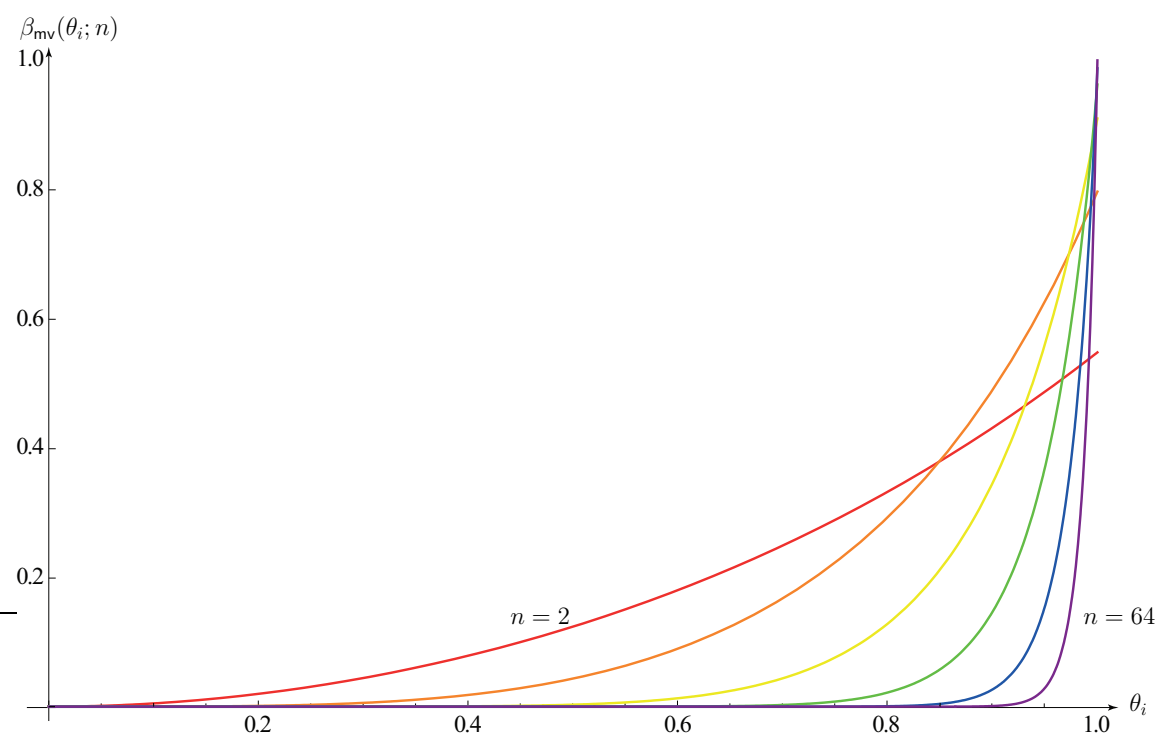

Figure 2: Equilibrium bidding functions for the all-pay auction under mean-variance preferences for uniformly distributed valuations, $\nu=1 / 4$, and $n \in\{2,4,8,16,32,64\}$ players, respectively (sorted in the colours of the rainbow from red to violet).

Corollary 1. In the symmetric equilibrium described in proposition 1 ,

1. low types decrease their bids and high types increase their bids relative to the case with a lower number of bidders;

2. the type $\hat{\theta}$ who issues the same bid under mean-variance and risk-neutral von Neumann-Morgenstern preferences shifts to the right

as the number of participating bidders $n$ increases.

Proof. Consider the derivative of (6) with respect to $n$

$$
\theta f(\theta) F(\theta)^{n-3}\left[(1-\theta \nu) F(\theta)(1-\kappa)+2 \theta \nu F(\theta)^{n}(1-2 \kappa)\right]
$$

where $\kappa=-(n-1) \log (F(\theta))$. Notice that $\log (F(\theta)) \leq 0$ and $\log (F(\theta))$ is strictly increasing in $\theta$ with $\log (F(\theta)) \rightarrow-\infty$ as $\theta$ approaches the lower bound of the support of its distribution and $\log (F(\theta)) \rightarrow 0$ as $\theta$ approaches the upper bound of the support of its distribution. Therefore, for sufficiently small $\theta,(10)$ becomes negative. Similarly, for $\theta$ sufficiently large, (10) is positive.

\footnotetext{
${ }^{21}$ Fibich et al. (2006) also have numerical results which do not rely on this assumption.
} 
Corollary 1 shows that the comparative statics of equilibrium bidding in the number of participants in an all-pay auction qualitatively do not differ whether bidders are risk neutral or symmetrically variance-averse. However, as shown in the second part of the corollary, the convergence to a distribution where only high types submit positive bids is faster the more variance-averse the bidders are.

So far, we have shown that in the all-pay auction consideration of variance aversion generates the commonly observed bidding behaviour, which is often attributed to risk aversion. Although our model suffices to predict such equilibrium bidding, it is much more tractable, specifically by yielding explicit equilibrium strategies under fairly general conditions on the distribution of players' types. Nevertheless, even rather simple distributions may yield expositionally unpleasant bidding functions (compare the "other distributions" examples below). For this reason, we will limit our discussion to the case of uniformly distributed player types for much of the remainder of this article. The relatively common assumption of uniformly distributed types allows us to exemplarily study a number of interesting extensions of our baseline model. Before proceeding, however, we will discuss our previous results under this particular assumption and provide bidding functions under different distributional assumptions for comparison.

\section{Examples for particular distributions}

We now briefly illustrate the findings of propositions 1 and 2 by means of specific examples of commonly used value distributions. For each case, we display symmetric, equilibrium bidding functions when players have mean-variance preferences and values are i.i.d. according to the specified distribution.

1. Consider the case of $n$ symmetrically variance-averse players when values are drawn from a uniform distribution over the interval $[0,1]$. In this case, the expression for the objective of a bidder with mean-variance preferences (1) simplifies to

$$
u_{i}\left(b, \theta_{i}\right)=\theta_{i}\left(\beta^{-1}(b)\right)^{n-1}\left(1-\nu \theta_{i}+\nu \theta_{i}\left(\beta^{-1}(b)\right)^{n-1}\right)-b
$$

which determines the symmetric equilibrium bidding functions as

$$
b^{*}=\beta\left(\theta_{i}\right)=\frac{n-1}{n} \theta_{i}^{n}+\nu\left(\frac{n-1}{n} \theta_{i}^{2 n}-\frac{n-1}{n+1} \theta_{i}^{n+1}\right) \text {. }
$$

Figure 3 compares this equilibrium bidding behaviour with that under standard risk-neutral von Neumann-Morgenstern preferences for two players. The Figure shows equilibrium bidding functions for the all-pay auction under risk-neutrality 
(dashed, $\left.\beta_{\mathbf{r n}}\left(\theta_{i}\right)=\theta_{i}^{2} / 2\right)$ and mean-variance preferences $(\nu=1 / 2$, solid). As seen in proposition 2 , the bidding behaviour of low-intermediate valuation players is more aggressive under expected payoff maximisation while high valuation players submit higher bids under mean-variance preferences. The two bidding functions coincide at $\hat{\theta}=2 / 3$ in this case.

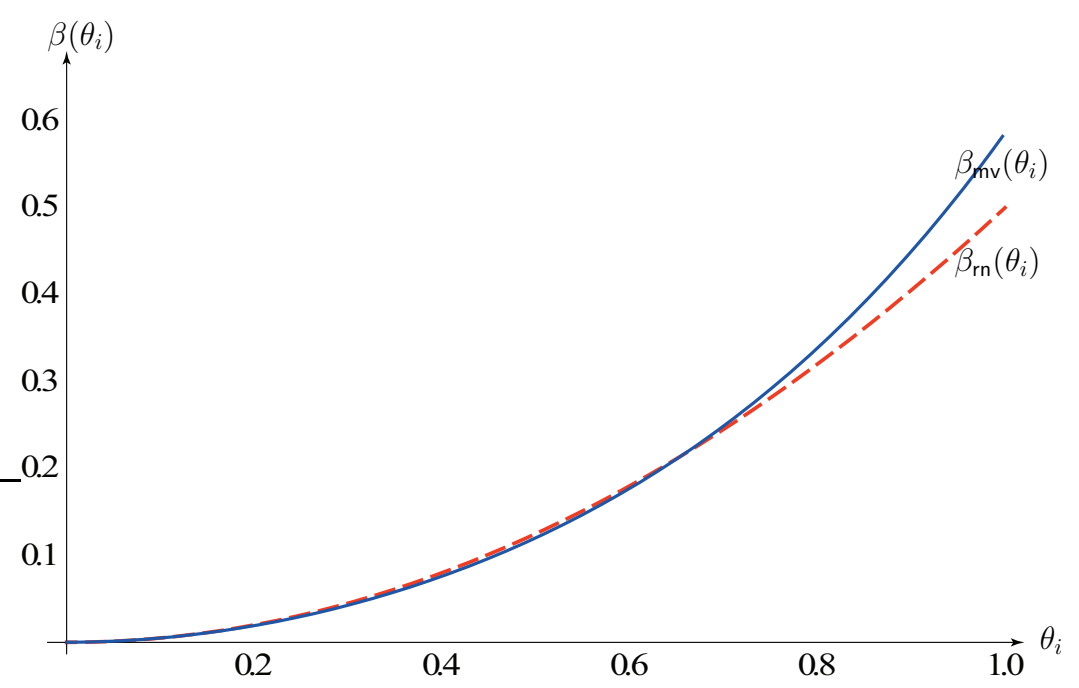

Figure 3: Equilibrium bidding functions for the two-player all-pay auction with independently and identically uniformly distributed valuations under risk-neutrality (dashed, $\left.\beta_{r n}\left(\theta_{i}\right)=\theta_{i}^{2} / 2\right)$ and mean-variance preferences $(\nu=1 / 2$, solid $)$.

2. For the Uniform distribution on $[0, U], U>0, F(\theta)=\theta / U$, the $n$-player bidding function is

$$
\beta\left(\theta_{i}\right)=\frac{(n-1) U\left(\theta_{i} / U\right)^{n}\left(\nu(n+1) U\left(\theta_{i} / U\right)^{n}-\theta_{i} \nu n+n+1\right)}{n(n+1)} .
$$

This is an example of type-supports extending beyond the unit interval (assumed elsewhere in the paper) which can be used to illustrate the relationship of the support of $F$ and the largest admissible $\nu$. From equation (9) follows that $\beta$ constitutes the symmetric equilibrium if and only if

$$
\nu<\frac{U^{n-1}}{(\theta+z)\left(U^{n-1}-2 z^{n-1}\right)}, \forall \theta \in[0, U], z \in(0, U)
$$

which is satisfied for any $n$ if $\nu \leq 1 /(2 U)$.

3. For the Power distribution on $[0,1], F(\theta)=\theta^{\alpha}, \alpha>0$, the two-player bidding function is

$$
\beta\left(\theta_{i}\right)=\alpha \theta_{i}^{\alpha+1}\left(\nu \theta_{i}\left(\frac{\theta_{i}^{\alpha}}{\alpha+1}-\frac{1}{\alpha+2}\right)+\frac{1}{\alpha+1}\right) .
$$


In this is case, optimality requires that $\nu<\left[(1+z)\left(1-2 z^{(n-1) \alpha}\right)\right]^{-1}$ for all $z \in(0,1)$.

4. Types distributed according to the Beta distribution with parameters $(2,2)$, $F(\theta)=\int_{0}^{\theta} u(1-u) d u / \int_{0}^{1} u(1-u) d u$, result in the symmetric two-player bidding function

$$
\beta\left(\theta_{i}\right)=\frac{\theta_{i}^{3}}{70}\left(3 \theta_{i}\left(\left(2 \theta_{i}\left(5\left(\left(7 \theta_{i}-20\right) \theta_{i}+14\right) \theta_{i}+14\right)-35\right) \nu-35\right)+140\right) .
$$

Here the critical valuation at which the objective is flat arises at $\hat{\theta}_{i}=1 / 16(\sqrt{33}+1)$ and the condition for the utility $u_{i}\left(b ; \hat{\theta}_{i}, \nu\right)$ to be non-decreasing at this critical type requires, approximately, that $\nu \leq 1.92$.

5. Finally, the Quadratic-U distribution, $F(\theta)=4(\theta-1 / 2)^{3}+4(1 / 2)^{3}$, gives rise to the equilibrium

$$
\begin{aligned}
\beta\left(\theta_{i}\right)=\frac{\theta_{i}^{2}}{14}\left(\left(\theta _ { i } \left(8\left(\theta_{i}\left(3\left(7 \theta_{i}-20\right) \theta_{i}+70\right)-42\right)\right.\right.\right. & \left.\left.\theta_{i}+105\right)-14\right) \theta_{i} \nu \\
& \left.+14\left(3 \theta_{i}-4\right) \theta_{i}+21\right) .
\end{aligned}
$$

Here, the highest type bids below or equal to 1 for any $\nu$ but weak monotonicity of $u_{i}\left(b ; \theta_{i}, \nu\right)$ requires that $\nu \leq 19 / 10$.

\section{Revenue valuation}

The classical reference for revenue valuation in winner-pay auctions under risk aversion is Holt (1980) who discusses a procurement setup. Revenue equivalence between the standard auction formats breaks down with risk averse bidders. While secondprice bidders maintain their dominant strategies of bidding their values, first-price competitors increase their bids with respect to the standard, risk-neutral case. This is due to the fact that raising one's bid in a first-price auction can be seen as partial insurance against losing. From a risk averse seller's point of view, the first-price auction is preferable to a second-price format because it exposes the seller to less revenue risk. ${ }^{22}$

Our objective in this section lies in the derivation of a series of concrete revenue ranking results based on two measures, expected revenue and revenue variance. We consider both a risk-neutral (i.e. expected revenue maximizing) seller and a seller who is variance averse.

\footnotetext{
22 For references, see Milgrom (2004, p. 123).
} 
Assumption. Valuations are independently and identically distributed with c.d.f.

$$
F\left(\theta_{i}\right)= \begin{cases}0 & \text { if } \theta_{i} \leq 0 \\ \theta_{i} & \text { if } \theta_{i} \in(0,1) \\ 1 & \text { if } \theta_{i}>1\end{cases}
$$

That is, valuations are uniformly distributed over $[0,1], \theta_{i} \sim U[0,1]$.

First notice that the seller's expected revenue R depends on the bidder's preferences. In the case of risk-neutral bidders, the seller expects to earn

$$
\mathbb{E}\left[\mathrm{R}_{\mathrm{rn}}\right]=n \int_{0}^{1} \frac{n-1}{n} \theta^{n} d \theta=\frac{n-1}{n+1} .
$$

If the bidders instead maximise a mean-variance function (1) with $\nu \in(0,1 / 2]$, then the seller can expect revenue

$$
\begin{aligned}
\mathbb{E}\left[\mathrm{R}_{\mathrm{mv}}\right] & =n \int_{0}^{1} \frac{n-1}{n} \theta^{n}+\nu\left(\frac{n-1}{n} \theta^{2 n}-\frac{n-1}{n+1} \theta^{n+1}\right) d \theta \\
& =\frac{n-1}{n+1}+\nu\left(\frac{n-1}{2 n+1}-\frac{n(n-1)}{(n+1)(n+2)}\right) .
\end{aligned}
$$

The revenue limit for $n \rightarrow \infty$ is $1-\nu / 2$.

Holding the number of players fixed, expected revenue is strictly increasing in $\nu$ for the two-players case and strictly decreasing in $\nu$ for all $n>2$. This is illustrated in figure 4 and summarised in corollary 2 .

Corollary 2. Consider symmetric equilibrium (5) of the $n$-player all-pay auction with mean-variance preferences. $\mathbb{E}\left[R_{m v}\right]$ is strictly increasing in $\nu$ if $n=2$ and strictly decreasing in $\nu$ if $n \geq 3$.

A consequence of this corollary is that the auctioneer's revenue in the (first-price) all-pay auction dominates that of the second-price auction for two bidders but is strictly lower for all other numbers of auction participants. The reason is that the dominantstrategy equilibrium revenue of a second-price auction is invariant with respect to the bidders' variance aversion $\nu$ and, hence, second-price revenue is given by (14). To see this more formally, consider a bidder's mean-variance optimisation problem in the second-price auction

$$
\begin{aligned}
\max _{b} & \int_{0}^{\beta^{-1}(b)}\left(\theta_{i}-\beta\left(\theta_{j}\right)\right) d \theta_{j} \\
-\nu & \left(\int_{0}^{\beta^{-1}(b)}\left(\left(\theta_{i}-\beta\left(\theta_{j}\right)\right)-\mu\right)^{2} d \theta_{j}+\int_{\beta^{-1}(b)}^{1}(0-\mu)^{2} d \theta_{j}\right)
\end{aligned}
$$


Supplying the candidate $\beta\left(\theta_{i}\right)=\theta_{i}$ and $\beta^{-1}(b)=b$ gives the first-order condition

$$
\left(b-\theta_{i}\right)\left(\nu^{2}\left(b\left(b-2 \theta_{i}-1\right)+\theta_{i}\right)-1\right)=0
$$

in which the first term is solved by the equilibrium $\beta\left(\theta_{i}\right)=b=\theta_{i}$. (The second term only gives solutions outside of $[0,1]$.)

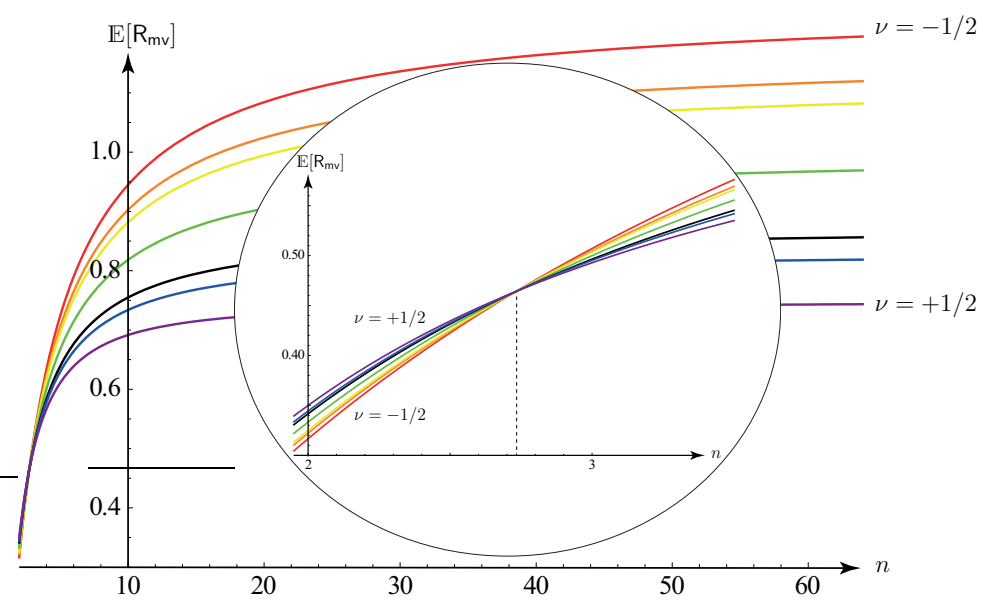

Figure 4: Revenue for $\nu \in\left\{-\frac{1}{2},-\frac{1}{3},-\frac{1}{4}, 0, \frac{1}{4}, \frac{1}{3}, \frac{1}{2}\right\}$ players, respectively (sorted in the colours of the rainbow from red to violet) and $\nu=0$ in black for $n \in[2,64]$. The analysis of variance seeking behaviour (i.e., negative $\nu$ ) is presented in section 5.3.

If the seller himself also considers the revenue variance in addition to the revenue's mean, then his preference may be reversed. In the case of risk-neutral bidding, the seller's revenue variance is

$$
\mathbb{V}\left[\mathrm{R}_{\mathrm{rn}}\right]=n \int_{0}^{1}\left(\beta_{\mathrm{rn}}(\theta)-\frac{\mathbb{E}\left[\mathrm{R}_{\mathrm{rn}}\right]}{n}\right)^{2} d \theta=\frac{n(n-1)^{2}}{(2 n+1)(n+1)^{2}} .
$$

If bidders maximise the mean-variance function (1), the resulting revenue variance is

$$
\begin{aligned}
\mathbb{V}\left[\mathrm{R}_{\mathrm{mv}}\right]=n \int_{0}^{1}\left(\beta_{\mathrm{mv}}(\theta)-\frac{\mathbb{E}\left[\mathrm{R}_{\mathrm{mv}}\right]}{n}\right)^{2} d \theta \\
=\mathbb{V}\left[\mathrm{R}_{\mathrm{rn}}\right]+\frac{n(n-1)^{2}}{(2 n+1)^{2}}\left(\nu \frac{7+n(21-4 n(n-3))}{(1+n)^{2}(2+n)(1+3 n)}\right. \\
\left.\quad+\nu^{2} \frac{74+n(151+8 n(n-3)(n-1))}{(2+n)^{2}(3+2 n)(2+3 n)(1+4 n)}\right) .
\end{aligned}
$$

The ratio of $\mathbb{V}\left[R_{m v}\right] / \mathbb{V}\left[R_{r n}\right]$ is given by

$$
1+\frac{(7+(7-2 n) n) \nu}{(2+n)(1+3 n)}+\frac{(1+n)^{2}(74+n(151+8(n-3)(n-1) n)) \nu^{2}}{(2+n)^{2}(1+2 n)(3+2 n)(2+3 n)(1+4 n)} .
$$

Figure 5 shows for different values of $\nu$ how this ratio reacts to a change in the number of bidders $n$. With variance-averse bidders, (20) is greater than one and increasing in 
$\nu$ for $n=2,3,4$. For $n \geq 5$ the variance ratio is decreasing in $\nu$ and below one. With variance-seeking players $(\nu<0$, compare section 5.3) these observations are reversed. All intersections shown in figure 5 are between 4 and 5 players. These results are summarised in the following corollary.

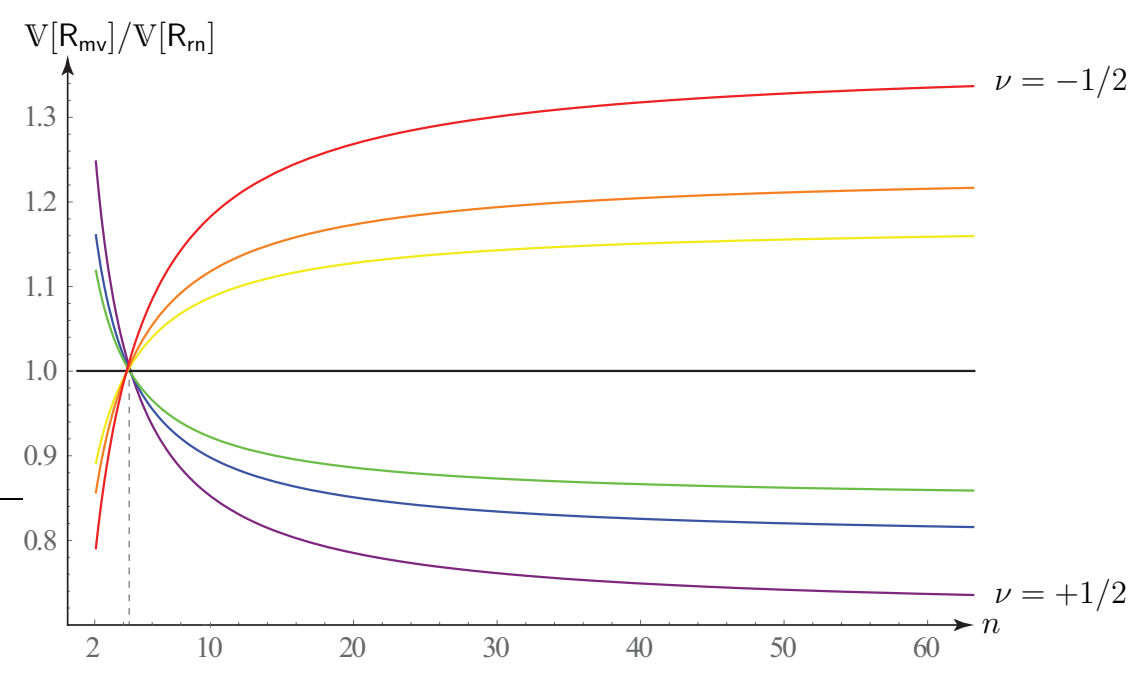

Figure 5: Seller's variance of revenue ratio for risk neutral bidders over variance averse bidders (20) for $\nu \in\left\{-\frac{1}{2},-\frac{1}{3},-\frac{1}{4}, 0, \frac{1}{4}, \frac{1}{3}, \frac{1}{2}\right\}$, sorted in the colours of the rainbow from red to violet and $\nu=0$ in black for $n \in[2,64]$.

Corollary 3.

\begin{tabular}{|c|c|c|c|}
\hline & $n=2$ & $n=3,4$ & $n \geq 5$ \\
\hline$\frac{\partial \mathbb{E}\left[R_{m v}\right]}{\partial \nu}$ & $>0$ & $<0$ & $<0$ \\
\hline$\frac{\partial \mathbb{V}\left[R_{m v}\right]}{\partial \nu}$ & $>0$ & $>0$ & $<0$ \\
\hline
\end{tabular}

Therefore, for $n=3,4$, both the expected revenue maximising and the varianceaverse sellers prefer to attract less variance-averse bidders. In all other cases, a variance-averse seller may prefer bidders with mean-variance preferences, where the exact ranking depends on the degree of the seller's variance aversion.

\section{Generalisations}

Starting out from our baseline model presented in section 2, we use this section to introduce and discuss a number of extensions that we consider particularly interesting. Our main motivation in this section is to illustrate that the introduction of meanvariance considerations into the standard auction analysis allows for simple analytical 
predictions even in relatively complex environments in which solutions based on full von Neumann-Morgenstern preferences seem entirely out of reach.

\subsection{Two asymmetric bidders}

In this article, we study the effects of variance aversion on bidding behaviour in allpay auctions. The standard analysis considers bidders who coincide in their objective, i.e., expected payoff maximisation. Our model, on the other hand, puts the players' objective in the focus of analysis, therefore it appears natural to study bidders whose objective functions differ in the extend to which payoff variance is taken into account. Our simple one-parameter model allows us to perform this exercise. This section presents our results on all-pay auctions between bidders who are asymmetric in terms of their risk preferences.

Consider the following two-players setup featuring asymmetric degrees of variance aversion $\nu_{i}$ where player $i \in\{1,2\}$ maximises

$$
u_{i}\left(\mu\left(b, \theta_{i}\right), \sigma^{2}\left(b, \theta_{i}\right)\right)=\mu\left(b, \theta_{i}\right)-\nu_{i} \sigma^{2}\left(b, \theta_{i}\right) .
$$

We consider the particular case of $\nu_{1}=0$ and $\nu_{2}=\nu \in(0,1 / 2)$, i.e., bidder 1 is riskneutral while bidder 2 is variance-averse. Correspondingly, player 1's and 2's objective functions are

$$
\begin{aligned}
& u_{1}\left(b_{1} ; \theta_{1}\right)=\beta_{2}^{-1}\left(b_{1}\right) \theta_{1}-b_{1}, \text { and } \\
& u_{2}\left(b_{2} ; \theta_{2}\right)=\beta_{1}^{-1}\left(b_{2}\right) \theta_{2}-\nu\left(\theta_{2}^{2} \beta_{1}^{-1}\left(b_{2}\right)\left(1-\beta_{1}^{-1}\left(b_{2}\right)\right)\right)-b_{2},
\end{aligned}
$$

respectively.

From an argument similar to the one used in a standard (risk-neutral) all-pay auction follows that the two bidding functions $\beta_{1}(\cdot)$ and $\beta_{2}(\cdot)$ must share the same support. Intuitively, in equilibrium, no player's type can submit a strictly higher bid than the other player's highest type as such a bid does not affect the payoff variance (the player wins with certainty), but strictly lowers the expected payoff. Maximising (22) together with the boundary conditions $\beta_{1}(0)=\beta_{2}(0)=0$ and $\beta_{1}(1)=\beta_{2}(1)$ yield the asymmetric equilibrium bidding strategies.

Proposition 3. The equilibrium of an asymmetric two-player all-pay auction with uniformly distributed types and player-specific variance-aversion parameters $\nu_{1}=0$ 


$$
\begin{aligned}
& \text { and } \nu_{2}=\nu \in(0,1 / 2] \text { is } \\
& \beta_{1}(\theta)=\frac{\log \left(1-\theta^{2} \nu+\theta \nu\right)}{2 \nu}-\frac{\theta^{2}}{\theta \nu(\theta-1)-1} \\
& +\frac{1}{2 \sqrt{\nu(4+\nu)}}\left(\log \left(1-\sqrt{\frac{4+\nu}{\nu}}\right)-\log \left(\frac{\sqrt{\frac{4+\nu}{\nu}}+\theta(4+\theta(\nu+\sqrt{\nu(4+\nu)}))-1}{\theta \nu(\theta-1)-1}\right)\right), \\
& \beta_{2}(\theta)=\frac{1}{2 \nu}\left[\theta \nu-1+\sqrt{1-2 \theta \nu+\theta^{2} \nu(4+\nu)}+\sqrt{\frac{\nu}{4+\nu}} \log \left(\sqrt{\frac{4+\nu}{\nu}}-1\right)\right. \\
& -\log \left(\frac{1}{2}\left(1-\theta \nu+\sqrt{1-2 \theta \nu+\theta^{2} \nu(4+\nu)}\right)\right) \\
& \left.-\sqrt{\frac{\nu}{4+\nu}} \log \left(\theta(4+\nu)-1+\sqrt{\frac{(4+\nu)\left(1-2 \theta \nu+\theta^{2} \nu(4+\nu)\right)}{\nu}}\right)\right] \text {. }
\end{aligned}
$$

Proof. The objective functions (22) yield the pair of first-order conditions

$$
\begin{aligned}
\frac{\partial u_{1}\left(\theta_{1}, b_{1}\right)}{\partial b_{1}} & =\frac{1}{\beta_{2}^{\prime}\left(\beta_{2}^{-1}\left(b_{1}\right)\right)} \theta_{1}-1=0 \\
& \Leftrightarrow \beta_{2}^{\prime}\left(\beta_{2}^{-1}\left(b_{1}\right)\right)=\theta_{1} \\
\frac{\partial u_{2}\left(\theta_{2}, b_{2}\right)}{\partial b_{2}} & =\frac{1}{\beta_{1}^{\prime}\left(\beta_{1}^{-1}\left(b_{2}\right)\right)}\left(\theta_{2}-\nu \theta_{2}^{2}\left(1-2 \beta_{1}^{-1}\left(b_{2}\right)\right)\right)-1=0 \\
& \Leftrightarrow \beta_{1}^{\prime}\left(\beta_{1}^{-1}\left(b_{2}\right)\right)-2 \nu \theta_{2}^{2} \beta_{1}^{-1}\left(b_{2}\right)=\theta_{2}-\nu \theta_{2}^{2} .
\end{aligned}
$$

In equilibrium, $b_{1}=\beta_{1}\left(\theta_{1}\right)$ and $b_{2}=\beta_{2}\left(\theta_{2}\right)$. Thus, we substitute $\beta_{1}^{-1}\left(b_{1}\right)=\theta_{1}$ into (24) to obtain

$$
\beta_{2}^{\prime}\left(\beta_{2}^{-1}(b)\right)=\beta_{1}^{-1}(b) .
$$

Taking the derivative of $\beta_{1}^{-1}(b)$ and applying (24) gives

$$
\beta_{1}^{\prime}\left(\beta_{1}^{-1}(b)\right)=\frac{\beta_{2}^{\prime}\left(\beta_{2}^{-1}(b)\right)}{\beta_{2}^{\prime \prime}\left(\beta_{2}^{-1}(b)\right)}
$$

where we use $b$ as variable from the joint support of $\beta_{1}(\cdot)$ and $\beta_{2}(\cdot){ }^{23}$ Substituting (27) and (26) into (25) yields the following second-order differential equation in $\beta_{2}$

$$
\frac{\beta_{2}^{\prime}\left(\beta_{2}^{-1}(b)\right)}{\beta_{2}^{\prime \prime}\left(\beta_{2}^{-1}(b)\right)}-2 \nu \theta^{2} \beta_{2}^{\prime}\left(\beta_{2}^{-1}(b)\right)=\theta-\nu \theta^{2} .
$$

This differential equation can be solved using the boundary condition $\beta_{2}(0)=0$ to obtain

$$
\begin{aligned}
\beta_{2}\left(\theta_{2}\right) & =\frac{1}{2 \sqrt{1+4 c} \nu}[\sqrt{1+4 c}(-1+\theta \nu+\sqrt{1+\theta \nu(-2+\theta \nu+4 c \theta \nu)}+\log (2)) \\
& +\log (1-\sqrt{1+4 c})-\sqrt{1+4 c} \log (1-\theta \nu+\sqrt{1+\theta \nu(-2+\theta \nu+4 c \theta \nu)}) \\
& \left.-\log \left(1-\nu\left(\theta+4 c \theta+\sqrt{1+4 c} \sqrt{\frac{1}{\nu^{2}}+\frac{\theta}{\nu}(-2+\theta \nu+4 c \theta \nu)}\right)\right)\right]
\end{aligned}
$$

23 The standard argument applies that in the two-player, all-pay auction the supports of both players' bidding functions coincide. 
for yet undetermined constant of integration $c$. In order to solve for the first player's bidding function, we solve (26) for

$$
\beta_{1}(\theta)=\beta_{2}\left(\left(\beta_{2}^{\prime}\right)^{-1}(\theta)\right)
$$

where

$$
\left(\beta_{2}^{\prime}\right)^{-1}(\theta)=\frac{\theta}{\nu\left(\theta-\theta^{2}+c\right)}
$$

Setting $\beta_{1}(1)=\beta_{2}(1)$ implies that the only possible value for the constant of integration is $c=1 / \nu$. Substituting this constant into $\beta_{2}\left(\theta_{2}\right)$, we obtain the pair of bidding functions (23). Monotonicity and global optimality can be verified numerically.

Figure 6 illustrates the equilibrium bidding functions in comparison to the equilibria of the corresponding symmetric two-player auctions.

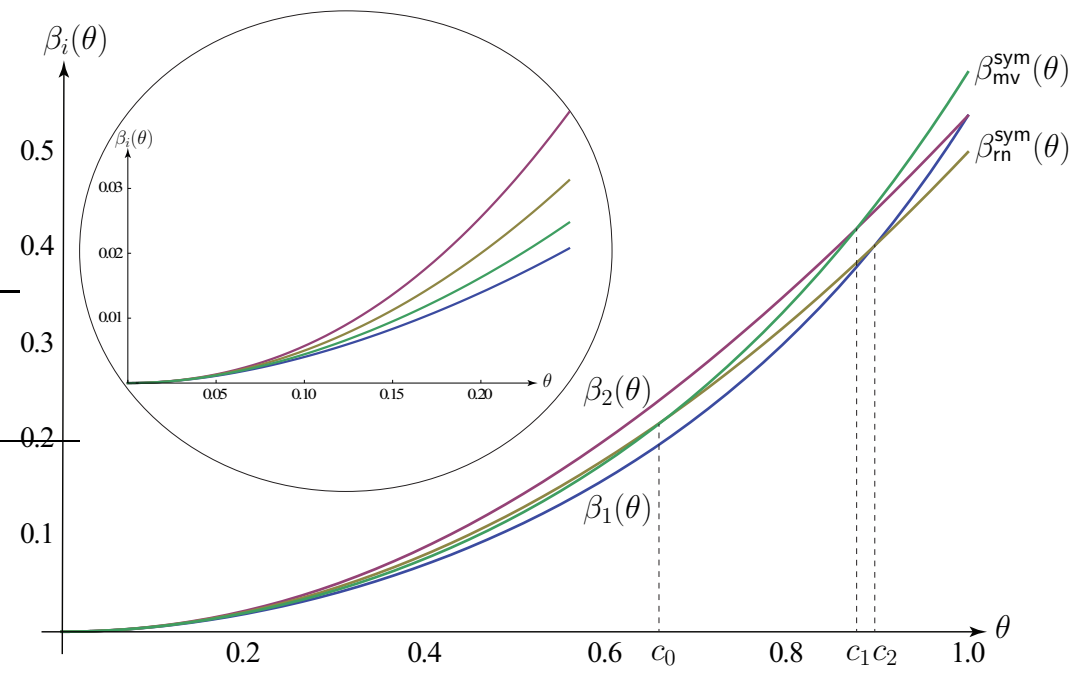

Figure 6: Comparison of asymmetric and symmetric bidding under mean-variance preferences for $\nu=1 / 2$.

As the figure shows, each positive risk-neutral player type bids less than the corresponding type of her variance-averse opponent. While the $\nu$-variance-averse, asymmetric bidder with bidding function $\beta_{2}(\cdot)$ always bids more than symmetric risk-neutral bidders $\beta_{\mathrm{rn}}^{\text {sym }}$, the asymmetric risk-neutral bidder with bidding function $\beta_{1}(\cdot)$ (competing with a variance-averse player) bids up to a cutoff-type $c_{2}$ below the symmetric riskneutral bidders and, for types higher than $c_{2}$, she bids above. Similarly, the asymmetric variance-averse bidder (competing with a risk-neutral bidder) bids up to a cutoff-type $c_{1}$ above the symmetric $\nu$-variance-averse bidders $\left(\beta_{\mathrm{mv}}^{\mathrm{sym}}\right)$ and bids below for types higher than $c_{1}$. Both properties are qualitatively similar to the single-crossing property with cutoff $\hat{\theta}=c_{0}$ from proposition 2 . The generally high bids of the variance-averse bidder cause low types of the risk-neutral bidder to bid less in comparison to their strategy when faced with risk-neutral opponents. High types of the risk-neutral bidder, on the other hand, increase their bid in reaction to their variance-averse opponent's strategy. 


\subsection{Exogenous shocks}

For the purpose of analysing the effect of the precision of signals, we extend the basic model analysed in section 3 with exogenous noise governed by the two elliptical distributions $W$ and $L$, i.e., distributions which are completely determined by their mean and variance. ${ }^{24}$ In a similar fashion to Esö \& White (2004) we consider the possibility that the final value of the object may further be influenced by an exogenous shock, $\varepsilon \sim W\left(0, \hat{\varepsilon}^{2}\right)$, which is distributed over some compact interval with mean zero and variance $\hat{\varepsilon}^{2}$. Similarly, a player's valuation of the state in which she does not win the object may be subject to another independently distributed exogenous shock $\delta \sim L\left(0, \hat{\delta}^{2}\right)$.

After realising their own (expected) valuations of the object, $\theta_{i}$, all players simultaneously submit their bids, $b_{i}, i \in \mathcal{N}$. The player with the highest bid receives the object and all players forgo their bids. Consider winning valuations distributed $\tilde{\theta}_{i} \sim W\left[\theta_{i}, \hat{\varepsilon}^{2}\right]$, $\hat{\varepsilon}^{2} \in[0,1]$. Similarly, we allow for the case that a player's valuation, if she does not win the object, is subject to another exogenous shock $\delta \sim L\left(0, \hat{\delta}^{2}\right)$. The exogenous shocks realise after the auction stage has ended and player $i$ 's payoff $\pi_{i}\left(b_{i}, b_{-i} ; \theta_{i}\right)$ is given by

$$
\begin{cases}\theta_{i}+\varepsilon-b_{i} & \text { if } b_{i}>b_{j} \forall j \neq i \\ \frac{1}{m}\left(\theta_{i}+\varepsilon\right)+\frac{m-1}{m} \delta-b_{i} & \text { if } i \in Q=\left\{j \in \mathcal{N} \mid b_{j}=\max _{k \in \mathcal{N}} b_{k}\right\}, m=|Q|, \\ \delta-b_{i} & \text { if } \exists j: b_{i}<b_{j} .\end{cases}
$$

Notice that neither of these exogenous noise variables have any effects on equilibrium bidding behaviour in the standard model of buyers with risk-neutral von NeumannMorgenstern utility, who simply maximise expected payoffs. In the following we discuss how bidding strategies of buyers with mean-variance preferences are affected by noisy signals. The player's objective changes from (1) into

$$
\begin{aligned}
& u_{i}\left(\mu\left(b, \theta_{i}\right), \sigma^{2}\left(b, \theta_{i}\right)\right)=\mu\left(b, \theta_{i}\right)-\nu\left(\sigma^{2}\left(b, \theta_{i}\right)+\hat{\varepsilon}^{2} F\left(\beta^{-1}(b)\right)^{n-1}\right. \\
& \left.+\hat{\delta}^{2}\left(1-F\left(\beta^{-1}(b)\right)^{n-1}\right)\right)
\end{aligned}
$$

for $\nu \in\left[0, \frac{1}{2}\right]$.

Inserting back the expressions for the mean and variance (4), and maximisation of the resulting objective yields the symmetric equilibrium.

\footnotetext{
${ }^{24}$ Elliptical distributions are a generalisation of the normal family containing, among others, the uniform, Student-t, Logistic, Laplace, symmetric stable, and Normal distributions. A detailed presentation of these distributions is available in Fang et al. (1987). Alternatively, if distributions are employed that are characterised by the first $n$ moments, $n>2$, the higher moments are assumed to be irrelevant to bidders endowed with mean-variance preferences.
} 
Proposition 4. A symmetric equilibrium in the environment with exogenous noise consists of the bidding functions

$$
\hat{\beta}_{m v}\left(\theta_{i}\right)=\left\{\begin{aligned}
0 & \text { if } \theta_{i} \leq \theta_{0} \\
\beta_{m v}\left(\theta_{i}\right)-\left(F\left(\theta_{i}\right)\right)^{n-1} \nu\left(\hat{\varepsilon}^{2}-\hat{\delta}^{2}\right) & \text { if } \theta_{i}>\theta_{0}
\end{aligned}\right.
$$

where $\beta_{m v}\left(\theta_{i}\right)$ is defined in (5) and the "cutoff type" $\theta_{0}$ is implicitly defined as the solution to ${ }^{25}$

$$
\begin{aligned}
& \theta_{0}\left(F\left(\theta_{0}\right)\right)^{n-1}-\int_{0}^{\theta_{0}}(F(\vartheta))^{n-1} d \vartheta \\
& -\nu \int_{0}^{\theta_{0}} \vartheta^{2}(n-1)(F(\vartheta))^{n-2} f(\vartheta)\left(1-2(F(\vartheta))^{n-1}\right) d \vartheta-\left(F\left(\theta_{0}\right)\right)^{n-1} \nu\left(\hat{\varepsilon}^{2}-\hat{\delta}^{2}\right)=0 .
\end{aligned}
$$

Proof. Inserting the expressions for the mean and variance (4) into the players' objective yields

$$
\begin{aligned}
u_{i}\left(b, \theta_{i}\right)= & \left.\left(F\left(\beta^{-1}(b)\right)\right)^{n-1}\left[\theta_{i}\left(1-\nu \theta_{i}+\nu \theta_{i}\left(F\left(\beta^{-1}(b)\right)\right)^{n-1}\right)-\nu \hat{\varepsilon}^{2}\right)\right] \\
& +\left(1-\left(F\left(\beta^{-1}(b)\right)\right)\right)^{n-1} \hat{\delta}^{2} \\
& -b
\end{aligned}
$$

The first-order condition for maximisation of this expression with respect to $b$ is

$$
\begin{gathered}
{\left[\theta_{i}\left(1-\nu \theta_{i}+2 \nu \theta_{i}\left(F\left(\beta^{-1}(b)\right)\right)^{n-1}\right)-\nu\left(\hat{\varepsilon}^{2}-\hat{\delta}^{2}\right)\right] \times} \\
(n-1)\left(F\left(\beta^{-1}(b)\right)\right)^{n-2} f\left(\beta^{-1}(b)\right) \frac{\partial \beta^{-1}(b)}{\partial b}=1 .
\end{gathered}
$$

In the symmetric equilibrium $b=\beta\left(\theta_{i}\right)$ for all $i \in \mathcal{N}$, this yields the first-order differential equation

$$
\begin{aligned}
\beta^{\prime}\left(\theta_{i}\right)= & \theta_{i}\left(1-\nu \theta_{i}+2 \nu \theta_{i}\left(F\left(\theta_{i}\right)\right)^{n-1}\right)(n-1)\left(F\left(\theta_{i}\right)\right)^{n-2} f\left(\theta_{i}\right) \\
& -(n-1)\left(F\left(\theta_{i}\right)\right)^{n-2} f\left(\theta_{i}\right) \nu\left(\hat{\varepsilon}^{2}-\hat{\delta}^{2}\right) .
\end{aligned}
$$

This differential equation is solved by the bidding function. As we restrict bids to be non-negative, the resulting bidding function is still invertible over the relevant region. Optimality requires the usual restriction of $\nu$.

Note that the bidding function (32) constitutes an equilibrium in both cases, whether a zero bid is interpreted as abstaining from the contest and hence results in a winning probability of zero, or the winner is determined through efficient tiebreaking (i.e., ties are broken in favour of the player with the highest valuation for 25 A closed form solution for the 'cutoff type' is generally unavailable. 
the prize) in the event that all players bid zero, which happens with strictly positive probability if $\hat{\varepsilon}^{2}-\hat{\delta}^{2}>0$ and $F$ has full support $[0,1]$. Which of these cases is more appropriate depends on the exact environment to be modelled.

Similarly to the common practice of normalising the valuation of the outside option to zero, (32) shows that there is a degree of freedom to normalise the variance of one of the two possible outcomes. In the remainder we therefore normalise $\hat{\delta}^{2} \equiv 0$ for simplicity.

Corollary 4. Introducing exogenous noise $\hat{\varepsilon}^{2}>0$ on the prize rotates the optimal bidding schedule down, causing low-type bidders to abstain from participating in the auction.

Consequently, it lies in the interest of an effort maximising contest designer to minimise $\hat{\varepsilon}^{2}-\hat{\delta}^{2}$, i.e., make signals about the prize as precise as feasible, while possibly keeping the loser's payoff uncertain.

\section{Example}

We conclude this section with our usual two-bidders example with uniformly distributed valuations. Consider the equilibrium bidding function

$$
b^{*}=\hat{\beta}\left(\theta_{i}\right)=\frac{\theta_{i}^{2}}{2}-\nu\left(\frac{\theta_{i}^{3}}{3}-\frac{\theta_{i}^{4}}{2}+\theta_{i} \hat{\varepsilon}^{2}\right) .
$$

The bidding behaviour this suggests for the case of a stochastic prize parametrised by $\hat{\varepsilon}^{2}=1 / 4$, is shown as dotted line in figure 7 . Consider now a case in which we auction two objects valued $\theta_{1}>\theta_{2}$ with exogenous prize variance $\hat{\varepsilon}^{1}>\hat{\varepsilon}^{2}$. If (full demand) bidders submit separate bidding functions for each object, then our model is able to predict cases where the bid for the high-value/high-risk object is below that of the low-value/low-risk object as exhibited by the dashed pair of bidding functions relative to their solid counterparts in figure 7 . In the context of allocation of resources amongst objects in a portfolio of research projects, this observation shows that a variance-averse manager may allocate fewer resources to a project with a relatively high expected return if the prediction of its value in case of a success is very noisy.

\subsection{Variance seeking}

Up to this point of our analysis we have focused on players who aim to avoid variance in their payoffs. Although risk aversion is a much more common assumption in economic studies, empirical studies have found risk seeking behaviour in multiple environments including investment games and inventive activities (see e.g., Åstebro, 2003; Jindapon 


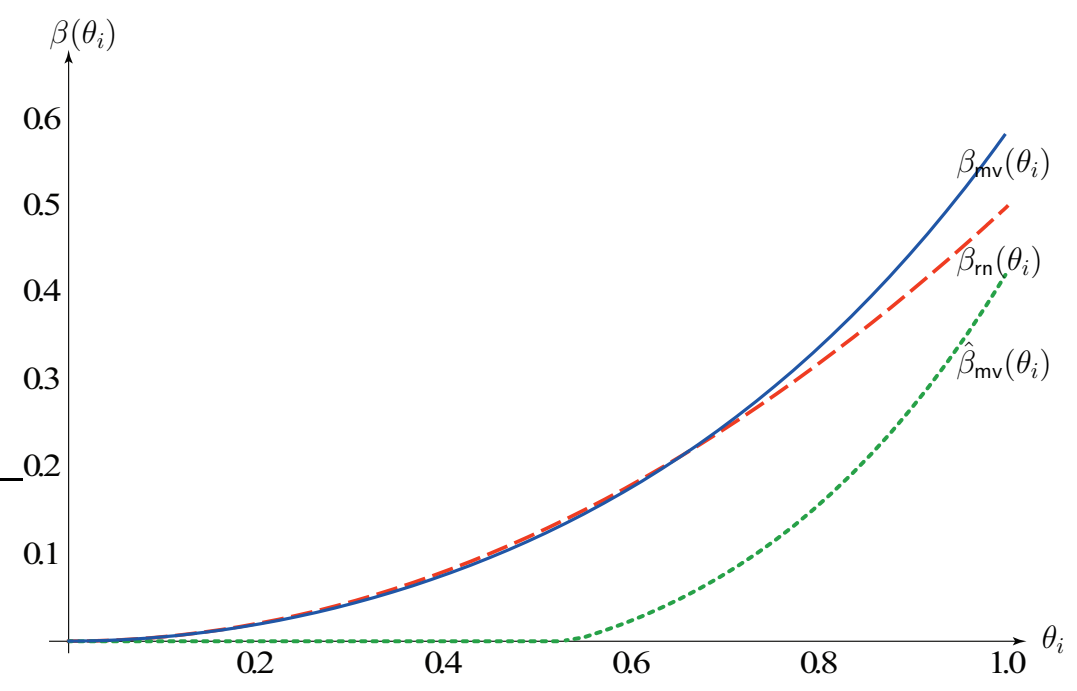

Figure 7: Equilibrium bidding functions for the all-pay auction with two players and uniformly distributed valuations under risk-neutrality (dashed, $\beta\left(\theta_{i}\right)=\theta_{i}^{2} / 2$ ) and mean-variance preferences $(\nu=1 / 2$, solid). The dotted bidding function results under mean-variance preferences if the prize itself is risky $\hat{\varepsilon}-\hat{\delta}=1 / 4$.

\& Yang, 2017). The topic of setting the right incentives for the alignment of managerial risk taking and investors' interests spans an entire literature. Agranov et al. (2014), for instance, show that subjects typically invest in a more risky manner when acting as managers in comparison to situations where individuals' personal funds are allocated. Therefore, it appears logical to consider variance seeking behaviour in the context of $R \& D$ races between firms if managers do not hold significant shares of the companies involved. For an analysis of how risk-taking influences a competition between fund managers whose compensation depends on how well they perform relative to each other, see Strack (2016).

In this section, we point out that the approach using mean-variance preferences is equally applicable when players are variance seeking and briefly discuss the effects of such preferences on our results in section 3. Variance seeking preferences are captured by a negative parameter, $\nu \in(-1 / 2,0)$, in our basic model $(1)$. Following the same analytical steps as before, we find that (5) still describes the symmetric equilibrium bidding functions when $n$ players have symmetric mean-variance preferences with variance seeking parameter $\nu \in(-1 / 2,0)$. Proposition 5 shows that in the equilibrium of the symmetric all-pay auction with variance seeking players low types bid more and high types bid less than their counterparts in the symmetric all-pay auction with risk neutral players.

Proposition 5 (Single-crossing II). In an all-pay auction with $n$ symmetric players, 
who each maximise (1) with $\nu \in(-1 / 2,0)$, (i) the strategies

$$
\beta_{m v}\left(\theta_{i}\right)=\beta_{r n}-\nu \int_{0}^{\theta_{i}} \vartheta^{2}(n-1)(F(\vartheta))^{n-2} f(\vartheta)\left(1-2(F(\vartheta))^{n-1}\right) d \vartheta
$$

constitute a symmetric equilibrium, and (ii) there exists a $\hat{\theta}$ in the support of $F$ such that $\beta_{m v}(\theta) \geq \beta_{r n}(\theta)$ for $\theta \leq \hat{\theta}, \beta_{m v}(\hat{\theta})=\beta_{r n}(\hat{\theta})$ and $\beta_{m v}(\theta)<\beta_{r n}(\theta)$ for $\theta>\hat{\theta}$.

Proof. The proof is the same as in section 3.

Figure 8 illustrates this observation graphically. It further shows that from a contest designer's point of view it is strongly beneficial to introduce even a small amount of exogenous noise when players have variance seeking preferences. In our model this effect can be achieved both by a higher degree of uncertainty about the value of the prize and by less uncertainty in the event of losing the contest.

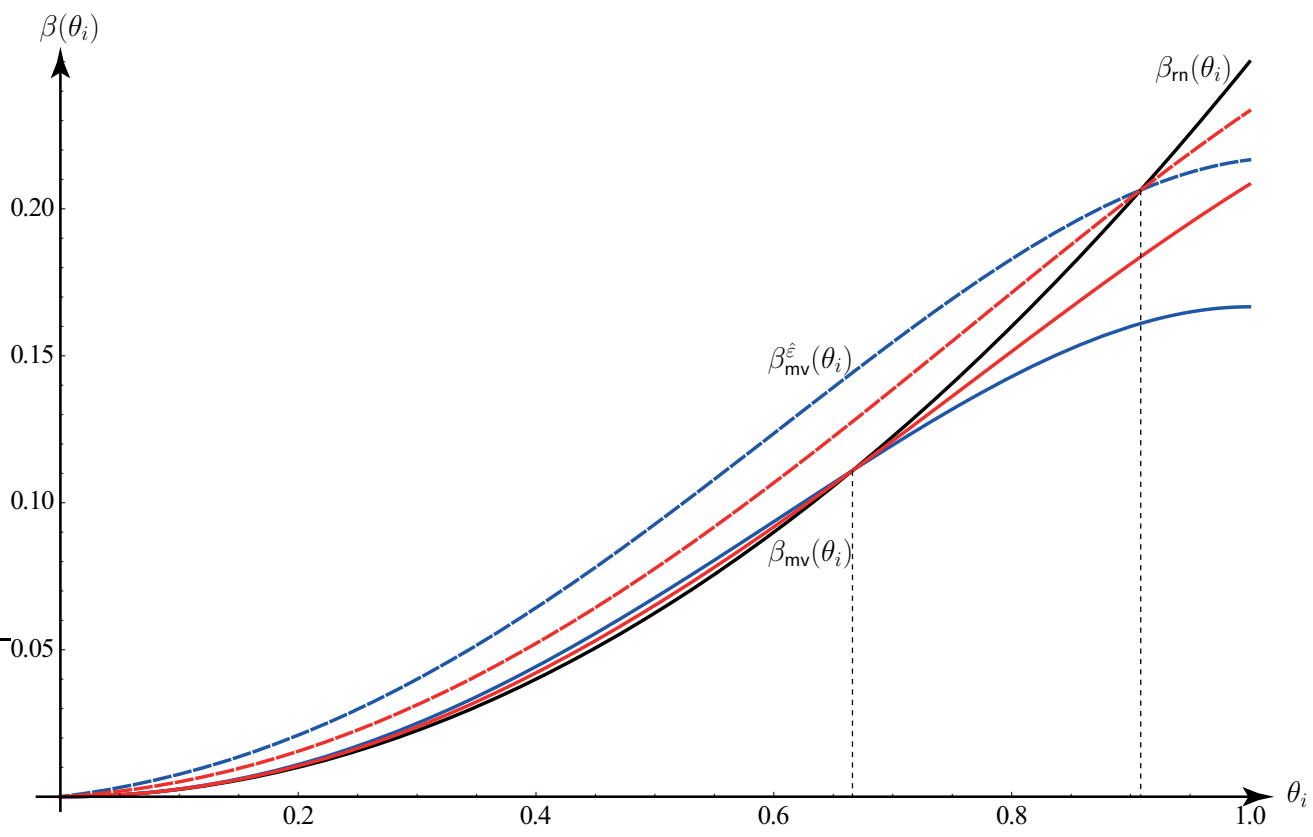

Figure 8: Equilibrium bidding functions for the all-pay auction under risk-neutrality (solid black, $\left.\beta_{\mathrm{rn}}\right)$ and risk-seeking behaviour $(\nu \in\{-1 / 4,-1 / 2\}$, solid, red and blue, resp.). The dotted bidding functions exhibit risky prizes $\hat{\varepsilon}-\hat{\delta}=1 / 10$.

Monotonicity of the bidding function (38) requires that

$$
\beta_{m v}^{\prime}\left(\theta_{i}\right)=\theta_{i}(n-1) F\left(\theta_{i}\right)^{n-3}\left(F\left(\theta_{i}\right)+2 \theta_{i} \nu F\left(\theta_{i}\right)^{n}-\theta_{i} \nu F\left(\theta_{i}\right)\right) f\left(\theta_{i}\right) \geq 0 .
$$

As previously discussed in section 3 , this is equivalent to

$$
F(\theta)^{n-1}>\frac{1}{2}-\frac{1}{2 \nu \theta}, \forall \theta \in[0,1]
$$


For negative $\nu$ this condition holds for any numbers of bidders $n$ and distribution of values $F(\cdot)$ if $\nu \geq-1$. However, equal to the case of variance aversion, we need to further restrict $\nu$ in order to guarantee that the identified strategy is a global maximizer. The condition

$$
F(z)^{n-1}>\frac{1}{2}-\frac{1}{2 \nu(\theta+z)}, \forall z \in(0,1)
$$

holds for any numbers of bidders $n$ and distribution of values $F(\cdot)$ if $\nu \in[-1 / 2,0]$.

\subsection{Type-dependent variance preferences}

In this section we further generalise our model to account for systematic differences in risk preferences. In section 5.1 we analyse bidding behaviour of two players who ex-ante differ in their risk attitudes. We take these variance aversion parameters as exogenously determined and assume that prize values are independently drawn from the same distribution. This model accurately captures bidding strategies when the mean-variance preference parameters are fixed. Contrary to this classical understanding of preferences, empirical and experimental studies suggest that the same player may indeed show different risk attitudes when facing different tasks and in particular when operating in different payoff intervals. In the spirit of Post \& Levy (2005), who test a model of locally risk-seeking agents whose risk attitudes differ in bear and bull markets, we generalise our model such that risk preferences systematically differ with a player's valuation of the prize. In order to study this case, we change the simple objective (1) to

$$
u_{i}\left(\mu\left(b, \theta_{i}\right), \sigma^{2}\left(b, \theta_{i}\right) ; \nu, \lambda, t\right)=\mu\left(b, \theta_{i}\right)-\nu\left(\theta_{i}-t\right)^{\lambda} \sigma^{2}\left(b, \theta_{i}\right)
$$

for $\lambda \in\{2 n+1 \mid n \in \mathbb{N}\} \cup\{0\}$ and reference point $t \in(0,1)$. Note that setting $\lambda=0$ takes us back to the case of type-independent mean-variance preferences as displayed in (1); in all other cases, for $\nu \in(0,1 / 2]$, types $\theta<t$ are variance-seeking while types $\theta>t$ are variance-averse. Type $\theta=t$ is risk neutral. A higher parameter $\lambda$ represents more pronounced differences in variance preference towards the extremes, while a lower parameter $\lambda$ corresponds to more uniformly increasing variance aversion. For the case where $\nu \in[-1 / 2,0)$, this interpretation is reversed.

Proposition 6. If players maximise (40), the symmetric equilibrium bidding strategy is

$\beta_{\nu, \theta}\left(\theta_{i} ; \nu, \lambda, t\right)=\frac{n-1}{n} \theta_{i}^{n}-\nu(n-1) t^{n+1}(-t)^{\lambda} \int_{0}^{\frac{\theta_{i}}{t}} x^{n}(1-x)^{\lambda}\left(1-2 t^{n-1} x^{n-1}\right) d x$. 
Proof. Taking the derivative of (40) with respect to player $i$ 's bid $b$, we obtain

$$
\begin{aligned}
\frac{\partial u_{i}\left(b, \theta_{i}\right)}{\partial b}= & \left(2 \theta_{i}^{2} \nu(n-1) \beta^{-1}(b)^{2 n}\left(\theta_{i}-t\right)^{\lambda}-\beta^{-1}(b)^{3} \beta^{\prime}\left(\beta^{-1}(b)\right)\right. \\
& \left.-\theta_{i}(n-1) \beta^{-1}(b)^{n+1}\left(\theta_{i} \nu\left(\theta_{i}-t\right)^{\lambda}-1\right)\right) /\left(\beta^{-1}(b)^{3} \beta^{\prime}\left(\beta^{-1}(b)\right)\right)
\end{aligned}
$$

in which we assume that the unknown function $\beta\left(\theta_{i}\right)$ is invertible. Setting zero and supplying the symmetric equilibrium condition $b=\beta\left(\theta_{i}\right)$, this simplifies to

$$
\beta^{\prime}\left(\theta_{i}\right)=(n-1) \theta_{i}^{n-1}\left(1+\left(\theta_{i}-t\right)^{\lambda} \theta_{i} \nu\left(2 \theta_{i}^{n-1}-1\right)\right) .
$$

A type-zero player still optimally bids zero. The first-order differential equation (43) is then solved for boundary condition $\beta(0)=0$ by (41).

This formulation allows for changes of variance-preferences based on endogenous, type-dependent considerations. Figure 9 shows representative bidding functions for positive and negatively type-related variance aversion in comparison to risk-neutral bidding.

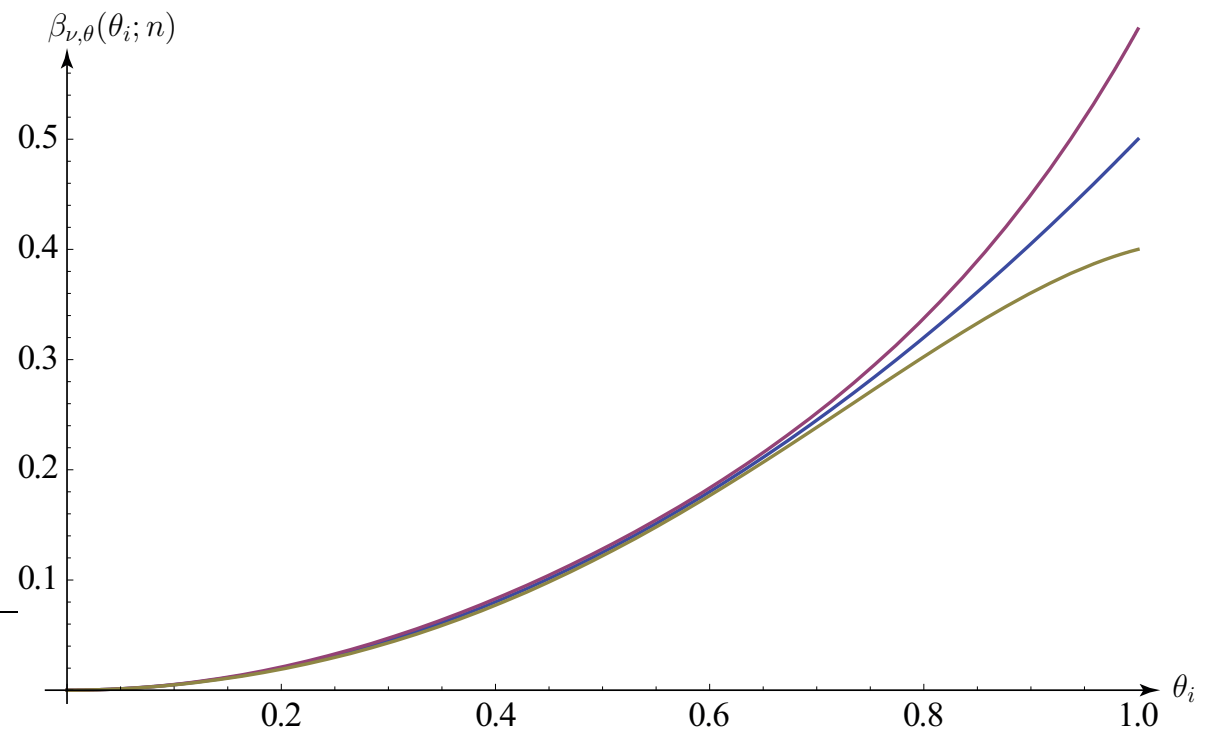

Figure 9: Two-player equilibrium bidding functions with type dependent variance aversion: $\beta_{\nu, \theta}(\theta ; 0,0,1 / 2)$ (blue), $\beta_{\nu, \theta}(\theta ; 1 / 2,1,1 / 2)$ (red), $\beta_{\nu, \theta}(\theta ;-1 / 2,1,1 / 2)$ (gold). The shown bidding functions do not intersect for $\theta>0$.

\section{Concluding remarks}

We present first results for the study of all-pay auctions if buyers and/or sellers are endowed with mean-variance preferences. We fully characterise the symmetric equilibrium bidding function of the all-pay auction with $n$ identical bidders when bidders 
maximise an additively separable function of their expected payoff and payoff variance. Our first proposition shows that consideration of mean-variance preferences suffices to derive the qualitative properties of the bidding function which Fibich et al. (2006) obtain in their analysis of a similar environment but considering any von NeumannMorgenstern utility function which entails risk aversion. These qualitative properties seem to correspond well to experimental data. As such, all our results based on this bidding function appear relevant even when payoff distributions are not fully characterised by their first two moments. Eisenhuth \& Grunewald (2017) observe similar effects on equilibrium bidding behaviour in their analysis of loss-averse players. Most standard models of loss-aversion are equivalent to our model of (linear) mean-variance preferences because the wedge between the winning and losing payoff is exogenously determined by a player's type in the all-pay auction.

In our model of mean-variance preferences, players choose a strategy that maximises the difference between their expected payoff and the payoff variance, which is weighted by a parameter, $\nu$, representing the players' degree of variance consideration. One major advantage of this approach is that we obtain closed form solutions for the bidding functions with just a single parameter representing variance aversion. This enables us to perform comparative statics. Furthermore, the specifically chosen functional form allows us to relax the standard assumption of identical preferences. We exemplary solve for the bidding functions in an all-pay auction with one expected payoff maximiser and one bidder with mean-variance preferences. In contrast to the symmetric equilibrium, we find that the mean-variance bidder of a given type always bids more than her risk neutral opponent of the same type. Although the analysis is only provided for the case of two bidders, the result would look similar if more general sets of $n_{1}$ risk neutral and $n_{2}$ mean-variance bidders were competing. Similarly, we conjecture that the qualitative findings from our benchmark case would carry over if the first bidder type was not risk neutral, but just less variance-averse than her opponent. Our first results and analysis of a contest with players who differ in their risk preferences open the door for further interesting studies. In particular, a model in which bidders have private information about their own risk preference appears interesting. The analysis of such a model, however, seems complex and is therefore outside the scope of this first investigation. Although we consider incomplete information about the opponents' degree of variance consideration in section 5.4, we reduce the dimension of private information by assuming a (known) particular function relating prize value and variance aversion parameter, $\nu_{i}=f\left(\theta_{i}\right)=\nu \cdot\left(\theta_{i}-t\right)^{\lambda}$.

Another crucial result of this article is the importance of bidders' risk preferences from the seller's perspective. Having obtained the (symmetric) equilibrium bidding 
function we consider effects of the number of bidders and their degree of variance aversion on revenue. Corollary 2 shows that the influence of variance aversion on expected revenue depends on the number of players. In particular, we find that considering $n \geq 3$ reverses the ranking found for the two-player case. This finding suggests that under risk-aversion the generalisation from the two-player case to the general case may not always be as intuitive as it is often the case under risk neutrality. The particular contest under consideration determines which fundamental parameters can be influenced by the organiser. Moreover, we show that the seller's own preference plays a crucial role when choosing the right number of participants with the preferential degree of variance consideration. This result is particularly important when the contest organiser is very influential. For example, a government whose main objective is to foster innovations - often measured by the number of registered patents - will optimally impact the innovative environment differently from one which is mainly interested in high value innovations. If start-ups are generally more risk-averse than large established companies, then different innovation related preferences will lead authorities to prefer different competitive structures.

With the exception of the analysis of bidding behaviour of $n$ ex-ante identical players, much of our analysis focuses on the case of valuations that are i.i.d. draws from the uniform distribution over $[0,1]$. The resulting simplification of otherwise lengthy expressions and the possibility to analytically obtain solutions has caused us to make this assumption. However, qualitatively similar results can be obtained for other standard distributions.

\section{Appendix}

Throughout the paper we use simple preferences of the form

$$
u_{i}\left(\mu\left(b, \theta_{i}\right), \sigma^{2}\left(b, \theta_{i}\right)\right)=\mu\left(b, \theta_{i}\right)-\nu_{i} \sigma^{2}\left(b, \theta_{i}\right), \text { with } \nu_{i} \in\left[-1 / 2,+^{1} / 2\right] .
$$

Consider now a more general symmetric utility formulation $u\left(\mu\left(b, \theta_{i}\right), \sigma^{2}\left(b, \theta_{i}\right)\right)$ with standard uniform valuations. Taking the derivative with respect to $b$ and making the usual simplifications gives the first-order condition

$$
\frac{\left(\beta^{\prime}(\theta)-\theta\right) u^{(1,0)}\left(\mu\left(b, \theta_{i}\right), \sigma^{2}\left(b, \theta_{i}\right)\right)+(2 \theta-1) \theta^{2} u^{(0,1)}\left(\mu\left(b, \theta_{i}\right), \sigma^{2}\left(b, \theta_{i}\right)\right)}{\beta^{\prime}(\theta)}=0 .
$$

Denoting by $\rho$ the ratio of marginal valuations of variance over mean and assuming 
this ratio to be constant, the first-order condition simplifies to ${ }^{26}$

$$
\beta^{\prime}(\theta)=\theta+\rho(1-2 \theta) \theta^{2} \text { in which } \rho=\frac{u^{(0,1)}(\mu(b), \sigma(b))}{u^{(1,0)}(\mu(b), \sigma(b))} .
$$

This differential equation is solved by the bidding function

$$
\beta(\theta)=\frac{1}{2} \theta^{2}+\rho \frac{2-3 \theta}{6} \theta^{3} .
$$

Standard arguments imply that $u^{(1,0)}>0$ and our interpretation of variance-aversion is that $u^{(0,1)}<0$. Since $\left(2 \theta-3 \theta^{2}\right) / 6 \geq 0$ if $\theta \leq 2 / 3$, this allows us to determine precisely which types of variance averse bidders bid in excess of their risk neutral counterparts. The equivalent to (47) for general symmetric distribution $F$ and $n \geq 2$ bidders is

$$
\beta(\theta)=(n-1) \int_{0}^{\theta} F(x)^{n-2}\left(\rho x\left(1-2 F(x)^{n-1}\right)+1\right) x f(x) d x .
$$

This illustrates that, although we choose a particularly simple form of preferences for presentational purposes, using more involved functional preference representations will leave many of our results qualitatively unaffected.

\section{References}

Agranov, M., Bisin, A., \& Schotter, A. (2014). An experimental study of the impact of competition for other people's money: the portfolio manager market. Experimental Economics, 17(4), 564-585. https://doi.org/10.1007/s10683-013-9384-6

Allais, M. (1953). Le comportement de l'homme rationnel devant le risque: critique des postulats et axiomes de l'École Américaine. Econometrica, 21(4), 503-46. https://doi.org/10.2307/1907921

Amann, E., \& Leininger, W. (1996). Asymmetric all-pay auctions with incomplete information: The two-player case. Games and Economic Behavior, 14, 1-18. https://doi.org/10.1006/game.1996.0040

Åstebro, T. B. (2003). The return to independent invention: Evidence of unrealistic optimism, risk seeking or skewness loving? Economic Journal, 113, 226-39. https://doi.org/10.1111/1468-0297.00089

Baron, D. P. (1977). On the utility theoretic foundations of mean-variance analysis. JF, 32, 1683-97. https://doi.org/10.1111/j.1540-6261.1977.tb03363.x

Barut, Y., Kovenock, D., \& Noussair, C. (2002). A comparison of multiple-unit allpay and winner-pay auctions under incomplete information. International Economic Review, 43, 675-708. https://doi.org/10.1111/1468-2354.t01-1-00031

\footnotetext{
${ }^{26}$ In case of utility function (44) we have that $u^{(1,0)}=1$ and $u^{(0,1)}=\nu_{i}$, i.e., the marginal valuations ratio is $\rho=\nu_{i}$.
} 
Blavatskyy, P. R. (2010). Modifying the mean-variance approach to avoid violations of stochastic dominance. Management Science, 56(11), 2050-2057. https://doi.org/10.1287/mnsc.1100.1224

Chen, Z. C., Ong, D., \& Segev, E. (2017). Heterogeneous risk/loss aversion in complete information all-pay auctions. European Economic Review, 95, 23-37. https://doi.org/10.1016/j.euroecorev.2017.03.002

Chipman, J. S. (1973). The ordering of portfolios in terms of mean and variance.

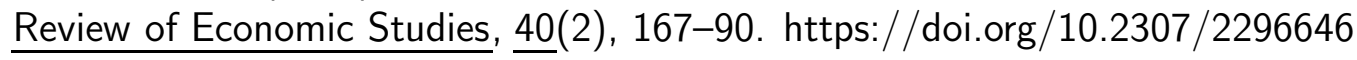

Chiu, H. (2010). Consistent mean-variance preferences. Oxford Economic Papers, 63 , 398-418. https://doi.org/10.1093/oep/gpq015

Cingottini, I., \& Menicucci, D. (2006). On the profitability of reducing competition in all-pay auctions with risk averse bidders. Economic Letters, 91, 260-6. https://doi.org/10.1016/j.econlet.2005.12.014

Cornes, R., \& Hartley, R. (2012a). Loss aversion in contests. University of Manchester, Economics Discussion Paper, EDP-1204.

Cornes, R., \& Hartley, R. (2012b). Risk aversion in symmetric and asymmetric contests. Economic Theory, 51, 247-75. https://doi.org/10.1007/s00199-009-0490-9

Coyle, B. T. (1992). Risk aversion and price risk in duality models of production: A linear mean-variance approach. American Journal of Agricultural Economics, 74 , 849-59. https://doi.org/10.2307/1243182

Dechenaux, E., Kovenock, D., \& Sheremeta, R. M. (2015). A survey of experimental research on contests, all-pay auctions and tournaments. Experimental Economics, 51(2), 315-50. https://doi.org/10.1007/s10683-014-9421-0

Eisenhuth, R., \& Grunewald, M. (2017). Auctions with loss averse bidders. International Journal of Economic Theory. (conditionally accepted)

Esö, P., \& Futó, G. (1999). Auction design with a risk averse seller. Economic Letters, 65, 71-74. https://doi.org/10.1016/S0165-1765(99)00115-9

Esö, P., \& White, L. (2004). Precautionary bidding in auctions. Econometrica, 72 , 77-92. https://doi.org/10.1111/j.1468-0262.2004.00478.x

Fang, K., Kotz, S., \& Ng, K. (1987). Symmetric multivariate and related distributions. London: Chapman \& Hall.

Fibich, G., \& Gavious, A. (2003). Asymmetric first-price auctions: A perturbation approach. Mathematics of Operations Research, 28(4), 836-52. https://doi.org/10.1287/moor.28.4.836.20510

Fibich, G., Gavious, A., \& Sela, A. (2004). Revenue equivalence in asymmetric auctions. Journal of Economic Theory, 115, 309-21. https://doi.org/10.1016/S00220531(03)00251-5

Fibich, G., Gavious, A., \& Sela, A. (2006). All-pay auctions with risk-averse players. International Journal of Game Theory, 34, 583-99. https://doi.org/10.1007/s00182-006-0034-5

Fu, Q., \& Lu, J. (2012). Micro foundations of multi-prize lottery contests: a per- 
spective of noisy performance ranking. Social Choice and Welfare, 38, 497-517. https://doi.org/10.1007/s00355-011-0542-5

Gneezy, U., Niederle, M., \& Rustichini, A. (2003). Performance in competitive environments: Gender differences. Quarterly Journal of Economics, 118(3), 1049-74. https://doi.org/10.1162/00335530360698496

Hanoch, H., \& Levy, M. (1970). A note on indifference curves and uncertainty. The Swedish Journal of Economics, 71(3), 206-8. https://doi.org/10.2307/3439370

Hillman, A. L., \& Samet, D. (1987). Dissipation of contestable rents by small numbers of contenders. Public Choice, 54, 63-82. https://doi.org/10.1007/BF00123805

Holt, C. A. (1980). Competitive bidding for contracts under alternative auction procedures. Journal of Political Economy, 88, 433-45. https://doi.org/10.1086/260878

Hu, A., Matthews, S., \& Zou, L. (2010). Risk aversion and optimal reserve prices in first and second-price auctions. Journal of Economic Theory, 145, 1188-202. https://doi.org/10.1016/j.jet.2011.10.005

Ingersoll, J. E. J. (1987). The theory of financial decision making. Rowman and Littlefield Publishing.

Ireland, N. (2004). Risk aversion and aggression in tournaments. University of Warwick, Working Paper. Retrieved from https://www2.warwick.ac.uk/fac/ soc/economics/staff/academic/ireland/risk_aversion_3.pdf

Jindapon, P., \& Yang, Z. (2017). Risk attitudes and heterogeneity in simultaneous and sequential contests. Journal of Economic Behavior and Organisation, 138, 69-84. https://doi.org/10.1016/j.jebo.2017.04.011

Johnstone, D., \& Lindley, D. (2013). Mean-variance and expected utility: The Borch paradox. Statistical Science, 28(2), 223-37. https://doi.org/10.1214/12-STS408

Kaplan, T., \& Zamir, S. (2012). Asymmetric first-price auctions with uniform distributions: analytic solutions to the general case. Economic Theory, 50, 269-302. https://doi.org/10.1007/s00199-010-0563-9

Kirkegaard, R. (2012). A mechanism design approach to ranking asymmetric auctions. Econometrica, 80, 2349-64. https://doi.org/10.3982/ECTA9859

Konrad, K. A., \& Schlesinger, H. (1997). Risk aversion in rentseeking and rent-augmenting games. Economic Journal, 107(445), 1671-83. https://doi.org/10.1111/j.1468-0297.1997.tb00074.x

Lazear, E. P., \& Rosen, S. (1981). Rank order tournaments as optimal labor contracts. Journal of Political Economy, 89, 841-64. https://doi.org/10.1086/261010

Leininger, W. (2000). Auction theory from an all-pay view: Buying binary lotteries. CESifo Working Paper Series, 382. Retrieved from http://www.cesifo-group .de/DocDL/cesifo_wp382.pdf

Levy, H., \& Markowitz, H. M. (1979). Aproximating expected utility by a function of mean and variance. American Economic Review, 69(3), 308-17. Retrieved from http://www. jstor.org/stable/1807366

Liu, L. (2004). A new foundation for the mean-variance analysis. European 
Journal of Operations Research, 158(1), 229-42. https://doi.org/10.1016/S03772217(03)00301-1

Lizzeri, A., \& Persico, N. (2000). Uniqueness and existence of equilibrium in auctions with a reserve price. Games and Economic Behavior, 30(1), 83-114. https://doi.org/10.1006/game.1998.0704

Markowitz, H. M. (1952). Portfolio selection. The Journal of Finance, 7(1), 77-91. https://doi.org/10.1111/j.1540-6261.1952.tb01525.x

Markowitz, H. M. (1959). Portfolio selection: Efficient diversification of investments (2nd ed.). New York, NY: John Wiley \& Sons Ltd.

Markowitz, H. M. (2014). Mean-variance approximations to expected utility. European Journal of Operations Research, 234(2), 346-55. https://doi.org/10.1016/j.ejor.2012.08.023

Markowitz, H. M., Reid, D. W., \& Tew, B. V. (1994). The value of a blank check. The Journal of Portfolio Management, 20(4), 82-91. https://doi.org/10.3905/jpm.1994.409480

Maskin, E., \& Riley, J. (1984). Optimal auctions with risk averse buyers. Econometrica, 52(6), 1473-1518. https://doi.org/10.2307/1913516

Maskin, E., \& Riley, J. (2000). Asymmetric auctions. Review of Economic Studies, 67, 413-38. https://doi.org/10.1111/1467-937X.00137

Matthews, S. (1987). Comparing auctions for risk averse buyers: A buyer's point of view. Econometrica, 55, 633-46. https://doi.org/10.2307/1913603

Mayer, J. (1987). Two-moment decision models and expected utility maximization. American Economic Review, $\underline{77}$, 421-30. https://doi.org/http://www.jstor.org/stable/1804104

Mermer, A. G. (2013). Contests with expectation-based loss-averse players. Working Paper, Tilburg University, October 25. Retrieved from http://hosting01.uc3m.es/semanal3/documents/Contests_with Expectation_Based_Loss_Averse_paper.pdf

Milgrom, P. (2004). Putting auction theory to work. Cambridge, UK: Cambridge University Press.

Nakamura, Y. (1970). Mean-variance utility. SSRN Discussion paper, \#2615290. https://doi.org/10.2139/ssrn.2615290

Niederle, M., \& Vesterlund, L. (2007). Do women shy away from competition? do men compete too much. Quarterly Journal of Economics, 122(3), 1067-101. https://doi.org/10.1162/qjec.122.3.1067

Noussair, C., \& Silver, J. (2006). Behavior in all-pay auctions with incomplete information. Games and Economic Behavior, 55, 189-206. https://doi.org/10.1016/j.geb.2005.01.005

Parreiras, S. O., \& Rubinchik, A. (2010). Contests with three or more heterogeneous agents. Games and Economic Behavior, 68(2), 703-715. https://doi.org/10.1016/j.geb.2009.09.007 
Pollatsek, A., \& Tversky, A. (1970). Theory of risk. Journal of Mathematical Psychology, 7, 540-53. https://doi.org/10.1016/0022-2496(70)90039-8

Post, T., \& Levy, H. (2005). Does risk seeking drive asset prices? A stochastic dominance analysis of aggregate investor preferences. Review of Financial Studies, 18(3), 925-53. https://doi.org/10.1093/rfs/hhi021

Qu, X. (2017). Subjective mean-variance preferences without expected utility. Mathematical Social Sciences, 87, 31-39. https://doi.org/10.1016/j.mathsocsci.2017.02.001

Riley, J. G., \& Samuelson, W. F. (1981). Optimal auctions. American Economic Review, 71, 381-92. Retrieved from http://www.jstor.org/stable/1802786

Robson, A. (2012). Contests between players with mean-variance preferences. Griffith Business School Discussion Paper, 2012-07. https://doi.org/10.2139/ssrn.21 $\overline{43008}$

Rothschild, M., \& Stiglitz, J. (1970). Increasing risk: I. a definition. Journal of Economic Theory, 2, 225-43. https://doi.org/10.1016/0022-0531(70)90038-4

Saha, A. (1997). Risk preference estimation in the nonlinear mean standard deviation approach. Economic Inquiry, 35, 770-82. https://doi.org/10.1111/j.14657295.1997.tb01963.x

Sahm, M. (2017). Risk aversion and prudence in contests. Economics Bulletin, 37(2), 1122-32. Retrieved from http://www.accessecon.com/Pubs/EB/2017/ Volume37/EB-17-V37-I2-P99.pdf

Sargent, T., \& Heller, S. (1987). Macroeconomic theory (2nd ed.). New York: Academic Press.

Savage, L. J. (1954). The foundations of statistics (2nd ed.). New York, NY: John Wiley \& Sons Ltd. https://doi.org/10.1002/nav.3800010316

Siegel, R. (2009). All-pay contests. Econometrica, 77, 71-92. https://doi.org/10.3982/ECTA7537

Simaan, Y. (1993). What is the opportunity cost of mean-variance investment strategies? Management Science, 39, 578-87. https://doi.org/10.1287/mnsc.39.5.578

Smith, J. L., \& Levin, D. (1996). Ranking auctions with risk-averse bidders. Journal of Economic Theory, 68, 549-61. https://doi.org/10.1006/jeth.1996.0031

Strack, P. (2016). Risk-taking in contests: The impact of fund-manager compensation on investor welfare. SSRN, Working paper. Retrieved from https://ssrn.com/ abstract $=2739177$

Tobin, J. (1958). Liquidity preference as behavior towards risk. Review of Economic Studies, 25(2), 65-86. https://doi.org/10.2307/2296205

Treich, N. (2010). Risk-aversion and prudence in rent-seeking games. Public Choice, 145(3-4), 339-49. https://doi.org/10.1007/s11127-009-9569-x

Tsiang, S. C. (1972). The rationale of the mean-standard deviation analysis, skewness preference, and the demand for money. American Economic Review, 62, 354-71. Retrieved from http://www. jstor.org/stable/1803382

von Neumann, J., \& Morgenstern, O. (1944). Theory of games and economic behavior. 
Princeton, NJ.: Princeton University Press. 